\title{
The functional geometry of local and horizontal connections in a model of $\mathrm{V} 1$
}

\author{
Paul C. Bressloff ${ }^{\mathrm{a}, *}$, Jack D. Cowan ${ }^{\mathrm{b}}$ \\ a Department of Mathematics, University of Utah, Salt Lake City, UT 84112, USA \\ ${ }^{\mathrm{b}}$ Department of Mathematics, University of Chicago, Chicago, IL 60637, USA
}

\begin{abstract}
A mathematical model of interacting hypercolumns in primary visual cortex (V1) is presented that incorporates details concerning the geometry of local and long-range horizontal connections. Each hypercolumn is modeled as a network of interacting excitatory and inhibitory neural populations with orientation and spatial frequency preferences organized around a pair of pinwheels. The pinwheels are arranged on a planar lattice, reflecting the crystalline-like structure of cortex. Local interactions within a hypercolumn generate orientation and spatial frequency tuning curves, which are modulated by horizontal connections between different hypercolumns on the lattice. The symmetry properties of the local and long-range connections play an important role in determining the types of spontaneous activity patterns that can arise in cortex.
\end{abstract}

(C) 2003 Published by Elsevier Ltd.

Keywords: Horizontal connections; Primary visual cortex; Orientation pinwheels; Spatial frequency; Mathematical modeling; Cortical dynamics; Neural pattern formation; Hallucinations

\section{Introduction}

One of the major simplifying assumptions in most large scale models of cortical tissue is that the interactions between cell populations are homogeneous and isotropic, that is, the pattern of connections is i nvariant under arbitrary rotations, translations and reflections in the cortical plane (for a review see [21]). However, these assumptions are no longer valid when the detailed microstructure of cortex is taken into account. This is exemplified by the functional and anatomical organization of primary visual cortex in cat and primates, which has a distinctly crystalline-like structure. Consider, for example, the distribution of cytochrome oxidase (CO) blobs [26,27]. These regions, which are about $0.2 \mathrm{~mm}$ in diameter and about $0.6 \mathrm{~mm}$ apart, coincide with cells that are more metabolically active and hence richer in their levels of $\mathrm{CO}$. Moreover, the distribution of $\mathrm{CO}$ blobs is correlated with a number of periodically repeating feature maps in which local populations of neurons re-

\footnotetext{
${ }^{*}$ Corresponding author.

E-mail addresses: bressloff@math.utah.edu (P.C. Bressloff), cowan@math.uchicago.edu (J.D. Cowan).
}

spond preferentially to stimuli with particular properties such as orientation, ocular dominance, and spatial frequency [5-8,33] (see Figs. 1-3). It has thus been suggested that the $\mathrm{CO}$ blobs could be the sites of functionally and anatomically distinct channels of visual processing [19,36,49,54].

Another manifestation of the crystalline-like structure of cortex is the distribution of singularities in the orientation preference map, as revealed, for example, by microelectrode recording $[28,29,31]$ and optical imaging [5-7]. These methods show that orientation preference changes continuously as a function of cortical location except at singularities or pinwheels, where the scatter or rate of change of differing orientation preference label is much higher, so that there is a weakening of orientation selectivity at the population level. Away from the pinwheels there exist approximate linear zones within which iso-orientation regions form parallel slabs. The linear zones tend to cross the borders of ocular dominance stripes at right angles, whereas the pinwheels tend to align with the centers of ocular dominance stripes. $\mathrm{CO}$ blobs are also located in the centers of ocular dominance stripes and have a strong association with about half of the orientation singularities. All of these features can be seen in the optical image shown in Fig. 1. 


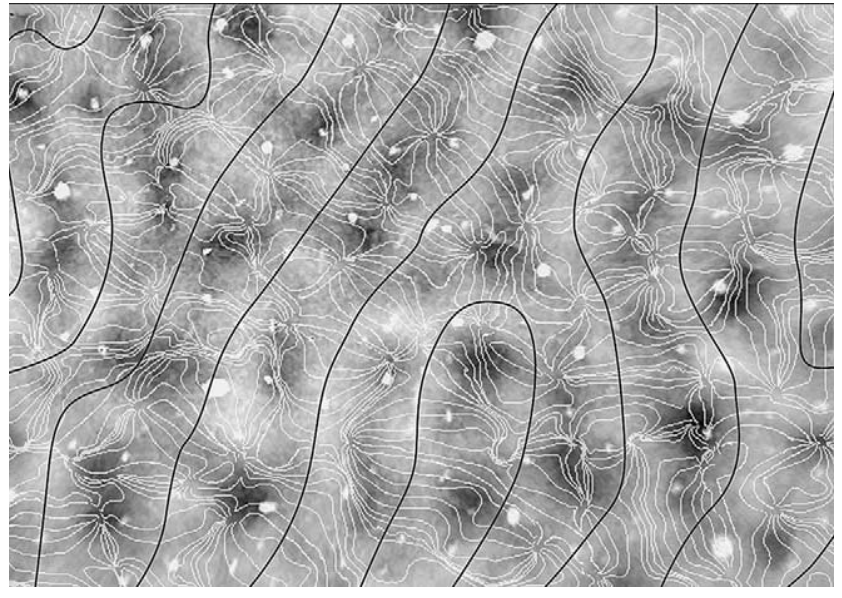

Fig. 1. Map of iso-orientation contours, ocular dominance boundaries and $\mathrm{CO}$ blob regions of Macaque V1. Redrawn from [5].

The distribution of spatial frequency preference across cortex is less clear than that of orientation preference. However 2-DG studies [54] established that there is a cortical map for spatial frequency in which low spatial frequency preference regions tend to coincide with $\mathrm{CO}$ blobs, and intermediate preference regions tend to avoid them. Fig. 2 shows some of these details. Intriguingly, recent optical imaging data concerning spatial frequency maps in cat suggest that those orientation singularities which do not coincide with $\mathrm{CO}$ blobs correspond to regions of high spatial frequency $[8,33]$. Fig. 3 , for example, shows that some iso-orientation singularities are surrounded by regions of low spatial frequency preference located in a background of higher spatial frequency preferences.

How does the crystalline-like structure of V1 manifest itself anatomically? Two cortical circuits have been fairly well characterized:

(1) There is a local circuit operating at sub-millimeter dimensions consisting of a mixture of intracortical excitation and inhibition. It has been suggested that such circuitry provides a substrate for the recurrent amplification and sharpening of the tuned response of cells to local visual stimuli. The best known example is the ring model of orientation preference and tuning $[4,50]$, in
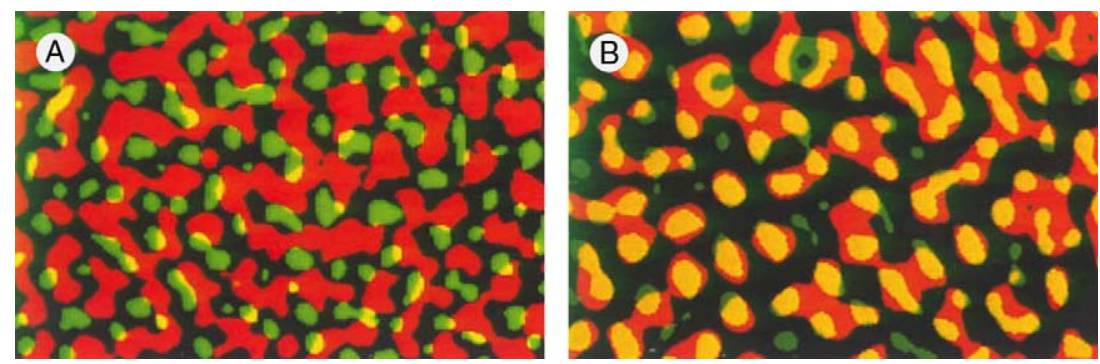

Fig. 2. Combined 2-DG and CO studies of V1. Regions of high DG uptake are color-coded red, regions of high CO staining are coded green, and regions of intersecting high DG and high cytox are coded yellow. Regions of low DG uptake and low cytox activity are coded black. In panel A the stimulus is an intermediate spatial frequency grating; in panel B it is a low spatial frequency one. It is apparent that in panel A the 2-DG uptake and the cytox stains are spatially separated, whereas in panel B they overlap. Redrawn from [54]. (For interpretation with reference to colour artwork the reader is referred to the web version of this article.)

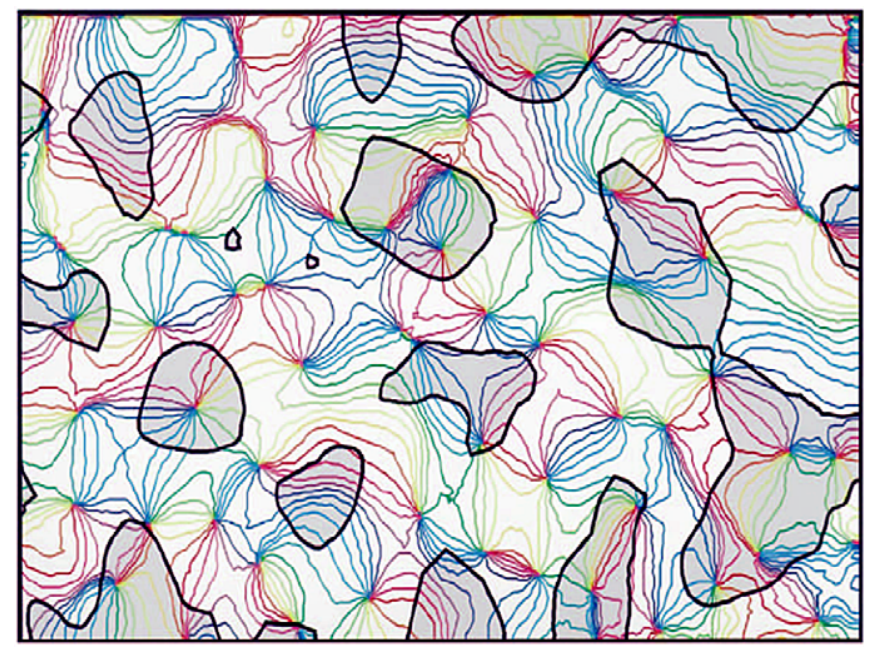

Fig. 3. Map of iso-orientation contours and low spatial frequency preferences in Cat V1. Filled regions correspond to low spatial frequencies, unfilled to high. Redrawn from [8]. 


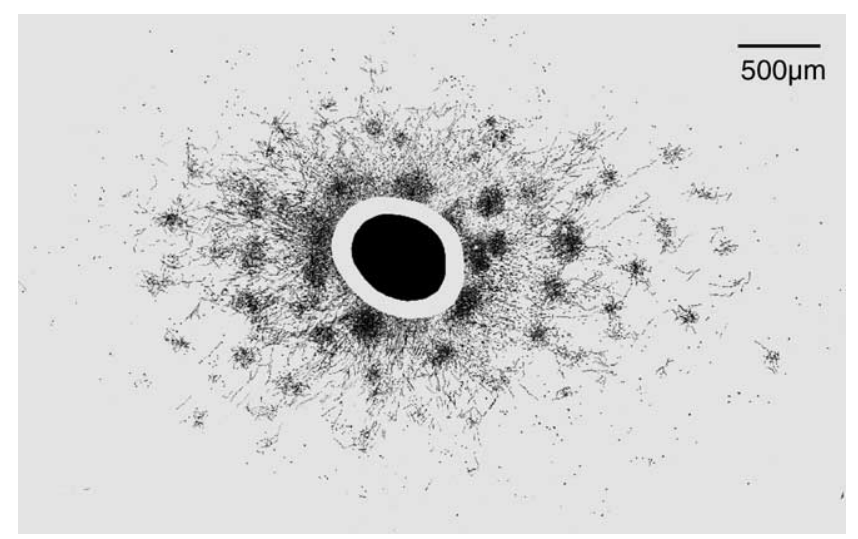

Fig. 4. Reconstruction of a tangential section through layers $2 / 3$ of macaque area V1, showing a CTB injection site and surrounding transported orthograde and retrograde label. Redrawn from Ref. [1].

which the local weights are assumed to vary as a function of the difference in orientation preference between the pre-synaptic and post-synaptic cells. One of the basic assumptions of the ring model is that the inhibitory connections are more broadly tuned with respect to orientation than the excitatory connections. This has recently received experimental support in the ferret [47]. The most likely source of such inhibition is the basket interneuron [37].

(2) The other circuit operates over a range of several millimeters and is mediated by the horizontally spreading axons of excitatory pyramidal neurons. These are illustrated in Fig. 4. By matching anatomical projections to optically imaged feature maps it has been found that the horizontal connections in superficial layers II/III (of cats and primates), which are broken into discrete terminal fields with a very regular size and spacing $[1,22$ 25,46], tend to emphasize links between neurons with similar functional properties $[9,38,58]$. Moreover, the horizontal connections tend to link blob to blob regions and interblob to interblob regions $[58,59]$.

We conclude from these various experimental observations that the local and long-range circuitry of V1 has a functional geometry, in the sense that the spatial distribution of connections is correlated with an underlying set of feature maps. The feature maps in turn suggest that V1 has a crystalline-like structure and can thus be partitioned into fundamental domains or hypercolumns $[30,34]$.

In this paper we present a large-scale model of V1 and its functional geometry, based on a lattice of interacting hypercolumns. Each hypercolumn is modeled as a network of interacting excitatory and inhibitory neural populations with orientation and spatial frequency preferences organized around a pair of pinwheels (see Section 2). The pinwheels are arranged on a planar lattice, reflecting the crystalline-like structure of cortex. Following our own recent work on orientation and spatial frequency tuning in a cortical hypercolumn $[13,14]$, the network topology of each hypercolumn is taken to be that of a sphere, with the poles of the sphere identified as low and high spatial frequency pinwheels respectively (see Section 3). A model for the horizontal connections linking different hypercolumns on the lattice is then introduced, which incorporates some anisotropy in the distribution of the patches (see Section 4). We then shown how the symmetry properties of the local and long-range connections play an important role in determining the types of spontaneous activity patterns that can arise in cortex (see Section 5). These patterns consist of spatially extended tuning surfaces for both orientation and spatial frequency.

We note that there are a number of reasons for being interested in cortical pattern formation. One direct application is to the theory of geometric visual hallucinations [20], which has been a major focus of our own recent work $[10,15,16]$. This theory proposes that the spontaneous activity patterns generated in cortex are seen as hallucinatory images in the visual field, whose spatial scale is determined by the range of horizontal connections and the retino-cortical map (see Section 6). However, we also believe that such work can provide insights into the normal functioning of the visual cortex. Indeed, recent optical imaging experiments have revealed that the cortex exhibits activity patterns in the absence of external visual stimulation that resemble those under conditions using single oriented stimuli [55]. Perhaps one way to understand the role of long range horizontal connections in the contextual processing of global stimuli is in terms of the way different stimuli excite the basic eigenmodes of cortex as revealed under hallucinatory conditions.

\section{The cortex as a lattice of hypercolumns}

On the basis of the 2-DG studies shown in Fig. 2, De Valois and De Valois [17] proposed the models of V1 hypercolumns shown in Fig. 5 for cat and macaque. These were modifications of the original icecube model of a hypercolumn introduced by Hubel and Wiesel [28]. In the case of the cat, orientation and spatial frequency preferences were taken to form orthogonal slabs suggestive of a linear feature map (see Fig. 5A). This picture was modified for macaque in order to include the $\mathrm{CO}$ blobs. In the macaque the observation that the $\mathrm{CO}$ blob regions respond preferentially to low spatial frequencies, suggested that spatial frequency increases radially away from the blobs. It is only relatively recently that $\mathrm{CO}$ blobs have also been found in cat V1 [43]. Optical imaging studies of the associated spatial frequency map $[8,32,33]$ have suggested further modifications to the original icecube model in which orientation and spatial frequency preferences are organized around a pair of 


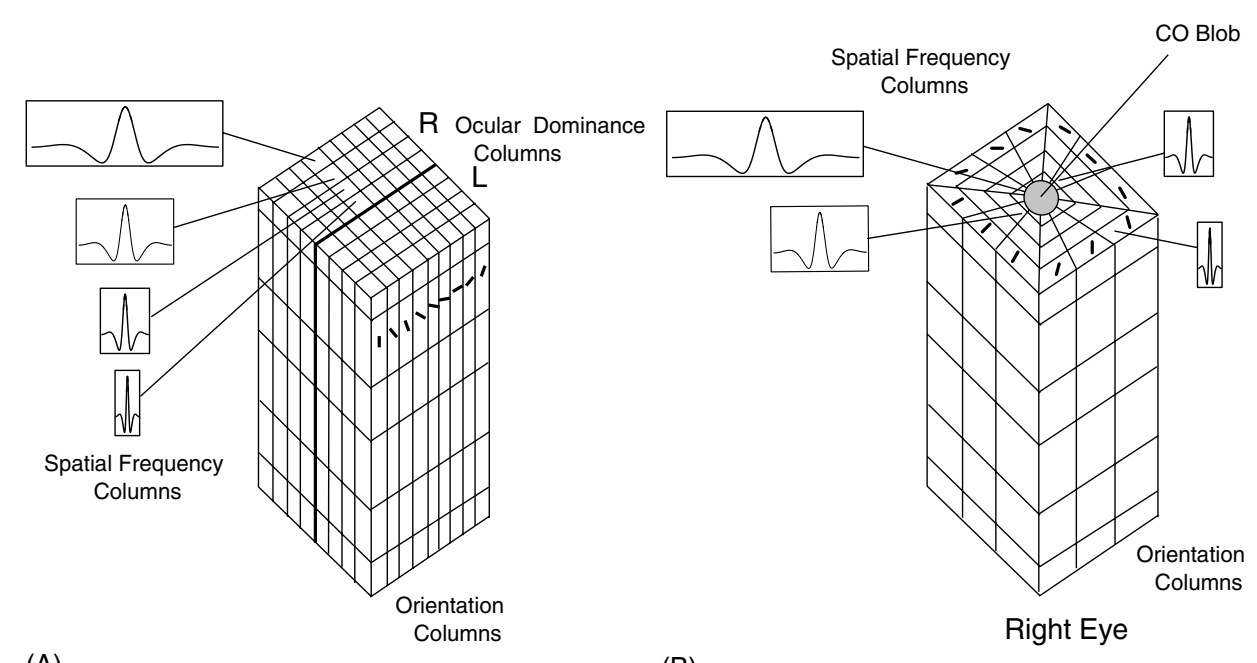

(A)

(B)

Fig. 5. (A) De Valois and De Valois' modified icecube model of a Cat V1 hypercolumn. (B) The modified icecube model with CO blobs for Macaque V1. Redrawn from [17].

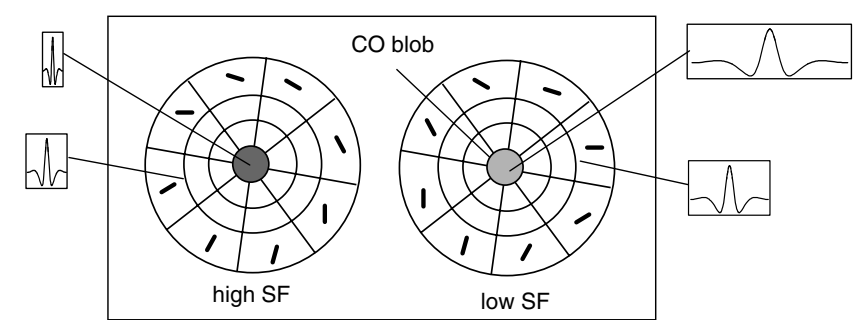

Fig. 6. Schematic diagram of a hypercolumn consisting of orientation and spatial frequency preferences organized around a pair of pinwheels.

orientation pinwheels corresponding to regions of high and low spatial frequencies respectively (see Fig. 6).

Although the partitioning of the cortex into hypercolumns has a degree of disorder, we assume that to a first approximation each hypercolumn is a fundamental domain $\Lambda_{\ell}$ of a planar lattice $\mathcal{L}$ of $\mathrm{CO}$ blobs with $\ell \in \mathcal{L}$. The lattice $\mathcal{L}$ is generated by two linearly independent vectors $\ell_{1}$ and $\ell_{2}$ :

$\mathcal{L}=\left\{\ell=m_{1} \ell_{1}+m_{2} \ell_{2}: m_{1}, m_{2} \in \mathbf{Z}\right\}$.

Let $\theta$ be the angle between the two basis vectors $\ell_{1}$ and $\ell_{2}$. We can then distinguish three types of lattice according to the value of $\theta$ : square lattice $(\theta=\pi / 2)$, rhombic lattice $(0<\theta<\pi / 2, \theta \neq \pi / 3)$ and hexagonal $(\theta=\pi / 3)$. Example fundamental domains for each of these lattices are shown in Fig. 13 (see Section 4). After rotation, the generators of the planar lattices are given in Table 1. Also shown are the generators of the dual lattice $\widehat{\mathcal{L}}$ satisfying $\hat{\ell}_{i} \cdot \ell_{j}=\delta_{i, j}$. The lattice spacing $L=\left|\ell_{1}\right|=\left|\ell_{2}\right|$ is taken to be the average width of a hypercolumn, that is, $L \approx 1 \mathrm{~mm}$.

Given the functional partitioning of cortex into a lattice of hypercolumns, we now construct a dynamical
Table 1

Generators for the planar lattices and their dual lattices

\begin{tabular}{lllll}
\hline Lattice & $L^{-1} \ell_{1}$ & $L^{-1} \ell_{2}$ & $L \hat{\ell}_{1}$ & $L \hat{\ell}_{2}$ \\
\hline Square & $(1,0)$ & $(0,1)$ & $(1,0)$ & $(0,1)$ \\
Hexagonal & $(1,0)$ & $\frac{1}{2}(-1, \sqrt{3})$ & $\left(1, \frac{1}{\sqrt{3}}\right)$ & $\left(0, \frac{2}{\sqrt{3}}\right)$ \\
Rhombic & $(1,0)$ & $(\cos \eta, \sin \eta)$ & $(1,-\cot \eta)$ & $(0, \csc \eta)$ \\
\hline
\end{tabular}

model of a single cortical layer. In order to proceed we need to specify both the internal structure of each hypercolumn and how the hypercolumns are coupled together on the lattice. First, suppose that a local patch of (excitatory and inhibitory) cells within a hypercolumn can be uniquely labelled by the pair $(\ell, P)$ where $\ell \in \mathcal{L}$ is the position of the hypercolumn on the lattice and $P$ represents a set of feature preferences corresponding to internal hypercolumn labels. Motivated by Fig. 6, we take $P=\{p, \phi\}$ where $p \in\left[p_{\min }, p_{\max }\right]$ denotes the spatial frequency preference and $\phi \in[0, \pi)$ the orientation preference of a cell. (For simplicity, we neglect ocular dominance here: this could be incorporated into the model by introducing an additional discrete label for left/right eye dominance). Typically, the bandwidth of a hypercolumn is between three and four octaves, that is, $p_{\max } \approx 2^{n} p_{\min }$ with $n=3.5$. This is consistent with the observations of Hubel and Wiesel [30], who found a two octave scatter of receptive field sizes at each cortical region they mapped. Let $a(\ell, P, t)$ denote the activity of the population $(\ell, P)$ at time time $t$, and suppose that $a$ evolves according to a Wilson-Cowan equation of the form [56,57]

$$
\begin{aligned}
\frac{\partial a(\ell, P, t)}{\partial t}= & -\alpha a(\ell, P, t)+h(\ell, P, t) \\
& +\sum_{\ell^{\prime} \in \mathcal{L}} \int w\left(\ell, P \mid \ell^{\prime}, P^{\prime}\right) \sigma\left(a\left(\ell^{\prime}, P^{\prime}, t\right)\right) D P^{\prime} .
\end{aligned}
$$


Here $\alpha$ is a decay rate (associated with membrane leakage currents or synaptic currents), $h(\ell, P, t)$ is an external input from the lateral geniculate nucleus (LGN), and the distribution $w\left(\ell, P \mid \ell^{\prime}, P^{\prime}\right)$ is the strength of connections from neurons at $\left(\ell^{\prime}, P^{\prime}\right)$ to neurons at $(\ell, P)$ with $D P^{\prime}$ an appropriately defined measure in feature space. The nonlinear firing-rate function $\sigma$ is assumed to be a smooth monotonically increasing function of the form $\sigma[z]$

$\sigma[z]=\frac{1}{1+\mathrm{e}^{-\gamma(z-\zeta)}}$

for constant gain $\gamma$ and threshold $\zeta$.

We would now like to decompose $w$ into local and long-range parts reflecting the distinct cortical circuits highlighted in Section 1. An attractive simplification from a computational viewpoint is to assume that the local connections mediate interactions within a hypercolumn whereas the patchy horizontal connections mediate interactions between hypercolumns. This suggests a decomposition of the form

$w\left(\ell, P \mid \ell^{\prime}, P^{\prime}\right)=\delta_{\ell, \ell^{\prime}} W\left(P \mid P^{\prime}\right)+\beta J^{\Delta}\left(\left|\ell-\ell^{\prime}\right|\right) W^{\Delta}\left(P \mid P^{\prime}\right)$,

where $W\left(P \mid P^{\prime}\right)$ and $W^{\Delta}\left(P \mid P^{\prime}\right)$ represent the dependence of the local and long-range interactions on the feature preferences of the pre- and post-synaptic cells, $\beta$ is a coupling parameter, and $J^{\Delta}(x)$ with $J(0)=0$ is a positive function that determines the variation in the strength of the long-range interactions with Euclidean distance on the lattice. However, as it stands, such a model does not take into account the fact that the local connections form a continuum across cortex so that, in particular, they couple neighbouring hypercolumns by forming connections across hypercolumn boundaries. In order to incorporate this additional coupling into the model, and to determine the detailed form of the local coupling function $W$, it would be necessary to specify in detail the spatial distribution of the orientation and spatial frequency maps (see Fig. 1). This is the approach taken by McLaughlin et al. [41], who have developed a computational model of a hypercolumn that explicitly incorporates details of the orientation map. However, this model does not consider spatial frequency tuning nor the effects of long range horizontal patchy connections [41]. An alternative approach is to consider a reduced model of a hypercolumn in which only a subset of neurons within a hypercolumn are explicitly represented, with the remaining cells acting as an effective background cell medium whose contribution is absorbed into the various weight functions of Eq. (2.4).

One possibility is to highlight an annular region of orientation selective cells (with fixed spatial frequency preference) around each orientation pinwheel and to take this as the reduced hypercolumn (see Fig. 7(A)). In this reduction scheme the distinction between high and
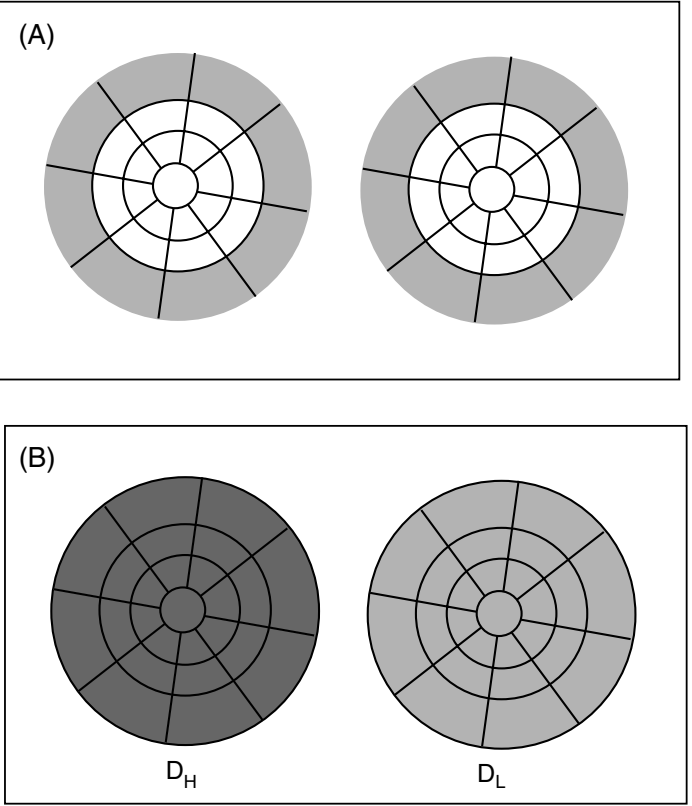

Fig. 7. Reduction of a hypercolumn. (A) Two identical rings of orientation selective cells. (B) Two non-identical discs $D_{H}$ and $D_{L}$ of orientation and spatial frequency selective cells, enclosing the high $(H)$ and low $(L)$ orientation pinwheels respectively.

low spatial frequency pinwheels is eliminated, leading to a renormalized lattice $\mathcal{L}^{*}$ with lattice spacing $L / 2$. Each hypercolumn is now labelled by the pair $(\ell, \phi), \ell \in \mathcal{L}^{*}$, and is taken to have the network topology of a ring. Thus the weight distribution (2.4) and the evolution equation (2.2) take the form

$$
\begin{aligned}
w\left(\ell, \phi \mid \ell^{\prime}, \phi^{\prime}\right)= & \delta_{\ell, \ell^{\prime}} W\left(\phi-\phi^{\prime}\right) \\
& +\beta J^{\Delta}\left(\left|\ell-\ell^{\prime}\right|\right) W^{\Delta}\left(\phi-\phi^{\prime}\right)
\end{aligned}
$$

and

$$
\begin{aligned}
\frac{\partial a(\ell, \phi, t)}{\partial t}= & -\alpha a(\ell, \phi, t)+\int_{0}^{\pi} W\left(\phi-\phi^{\prime}\right) \sigma\left(a\left(\ell, \phi^{\prime}, t\right)\right) \frac{\mathrm{d} \phi^{\prime}}{\pi} \\
& +\beta \sum_{\ell^{\prime} \neq \ell} J^{\Delta}\left(\left|\ell-\ell^{\prime}\right|\right) \int_{0}^{\pi} W^{\Delta}\left(\phi-\phi^{\prime}\right) \\
& \times \sigma\left(a\left(\ell^{\prime}, \phi^{\prime}, t\right)\right) \frac{\mathrm{d} \phi^{\prime}}{\pi}+h(\ell, \phi, t)
\end{aligned}
$$

with $W(\phi), W^{\Delta}(\phi)$ even, $\pi$-periodic functions of $\phi$. The qualitative behaviour of the distributions $W$ and $W^{\Delta}$ for
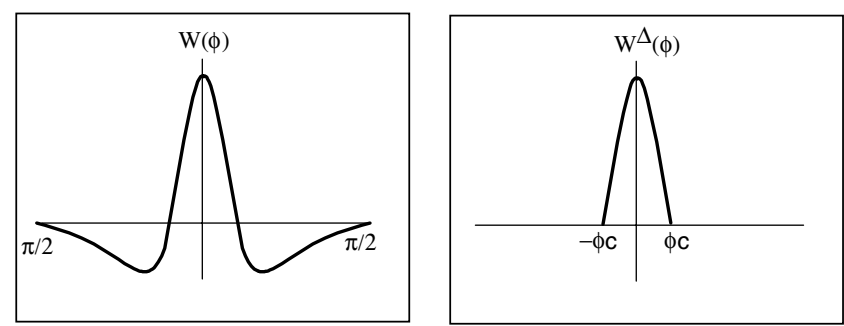

Fig. 8. Schematic diagram illustrating the qualitative behaviour of the local and long range horizontal weight distributions $W(\phi), W^{\Delta}(\phi)$. 
this model is summarized in Fig. 8. The local weight distribution $W(\phi)$ is assumed to consist of broadly tuned inhibition and more narrowly tuned excitation (the so-called "Mexican hat" function). On the other hand, $W^{\Delta}(\phi)$ is taken to be a positive, narrowly tuned distribution with $W^{\Delta}(\phi)=0$ for all $|\phi|>\phi_{c}$ and $\phi_{c} \ll \pi / 2$; the long-range connections thus link cells with similar orientation preferences.

Eq. (2.6) describes a lattice of coupled ring networks, each of which corresponds to the so-called ring model of orientation tuning $[4,50]$. As has been shown previously, local recurrent interactions within an isolated hypercolumn (idealized as a ring network) can amplify certain Fourier components of network activity leading to sharp orientation tuning curves, even when the LGN inputs are weakly biased. This amplification mechanism provides one explanation for the approximate contrast invariance of the tuned response [4,50]. Equations similar to (2.6) have subsequently been used to investigate how orientation tuning is modulated by long range horizontal interactions between hypercolumns $[16,18,35,42,51,52]$, as well as the role of horizontal connections in the spontaneous generation of cortical activity patterns underlying hallucinatory images $[10,15]$. However, it is clear that these models neglect important features of the internal structure of a hypercolumn illustrated in Fig. 6, namely the presence of a pair of orientation pinwheels [5-7,44] and associated variations in the spatial frequency selectivity of cells $[8,33]$. This motivates the alternative reduction scheme for a hypercolumn shown in Fig. 7(B), consisting of the union $D_{L} \cup D_{H}$ of two disc regions enclosing the low $(L)$ and high $(H)$ spatial frequency pinwheels respectively. Since the two discs are distinct, we recover the original lattice $\mathcal{L}$ and the pair of feature labels $P=\{p, \phi\}$. It remains to specify the network topology of the new hypercolumn model and the corresponding local and long-range weight distributions $W, W^{\Delta}$.

\section{Spherical model of a hypercolumn}

We begin by discussing the internal structure of the reduced hypercolumn model shown in Fig. 7(B). Suppose that interactions with the background cortical tissue leads to an effective identification or sewing together of the two disc boundaries $\partial D_{H}$ and $\partial D_{L}$. A well known result from topology is that the resulting space is a sphere. Identifying the north and south poles of the sphere with the low and high spatial frequency pinwheels leads to our so-called spherical model of a cortical hypercolumn $[13,14]$ (see Fig. 9). It is important to emphasize that the spherical topology is a mathematical idealization of the effective pattern of local interactions within a hypercolumn, and does not correspond to the actual distribution of cells in the cortical plane. It turns out that representing the hypercolumn as a sphere has a

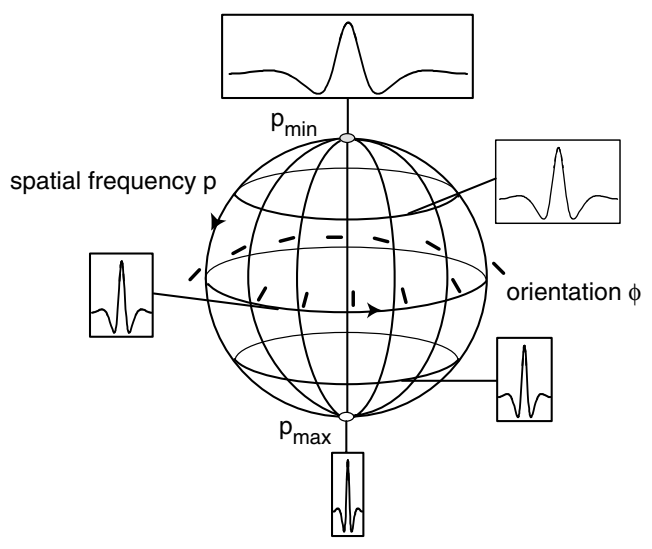

Fig. 9. Spherical network topology. Orientation and spatial frequency labels are denoted by $(\phi, p)$ with $0 \leqslant \phi<\pi$ and $p_{\min } \leqslant p \leqslant p_{\max }$.

number of desirable consequences [13,14]. In particular, the associated dynamical model reproduces a number of experimental observations regarding correlations between spatial frequency and orientation tuning curves $[33,39,40]$. One particular example is the reduction in orientation selectivity at high and low spatial frequency pinwheels.

Let $(\theta, \phi)$ to be the angular coordinates on the sphere with $\theta \in[0, \pi), \phi \in[0, \pi)$ then $\theta$ determines the spatial frequency preference $p$ according to

$\theta \equiv \mathscr{2}(p)=\pi \frac{\log \left(p / p_{\min }\right)}{\log \left(p_{\max } / p_{\min }\right)}$.

That is, $\theta$ varies linearly with $\log p$. This is consistent with experimental data that suggests a linear variation of $\log p$ with cortical separation [33]. Given a spherical topology, it is natural to construct a local weight distribution that is invariant with respect to coordinate rotations of the sphere, that is, the symmetry group $\mathbf{O}(3)$. This rotational symmetry, which generalizes the $\mathbf{O}(2)$ circular symmetry of the ring model, implies that the pattern of connections within the hypercolumn depends only on the relative distance of cells on the sphere as determined by their angular separation along geodesics or great circles. That is, given two points on the sphere $(\theta, \phi)$ and $\left(\theta^{\prime}, \phi^{\prime}\right)$ their angular separation $\alpha$ is

$\cos \alpha=\cos \theta \cos \theta^{\prime}+\sin \theta \sin \theta^{\prime} \cos \left(2\left[\phi-\phi^{\prime}\right]\right)$.

This suggests that the simplest non-trivial form for the local weight distribution $W\left(P \mid P^{\prime}\right)$ with $P=(\theta, \phi)$ and $P^{\prime}=\left(\theta^{\prime}, \phi^{\prime}\right)$ is

$$
\begin{aligned}
W\left(P \mid P^{\prime}\right)= & \widetilde{W}_{0}+\widetilde{W}_{1}\left(\cos \theta \cos \theta^{\prime}+\sin \theta \sin \theta^{\prime}\right. \\
& \left.\times \cos \left(2\left[\phi-\phi^{\prime}\right]\right)\right) .
\end{aligned}
$$

The associated integration measure on the sphere is then $D P=\sin \theta \mathrm{d} \theta \mathrm{d} \phi / 2 \pi$. In Fig. 10 we plot $W\left(P \mid P^{\prime}\right)$ as a function of $(\theta, \phi)$ for $\theta^{\prime}=\theta, \phi^{\prime}=0$ and $\widetilde{W}_{1}>\widetilde{W}_{0}$. It can be seen that away from the pinwheels (poles of the sphere at $\theta=0, \pi)$, cells with similar orientation excite 

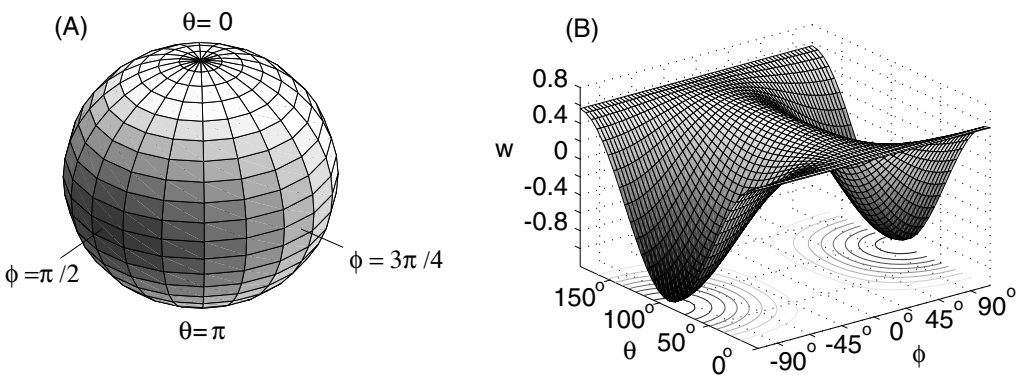

Fig. 10. Two dimensional plot of $W\left(\theta, \phi \mid \theta^{\prime}, \phi^{\prime}\right)$ given by the $\mathbf{O}(3)$-invariant weight distribution (3.3) with $\widetilde{W}_{0}=-1$ and $\widetilde{W}_{1}=1$. We set $\phi^{\prime}=0, \theta^{\prime}=\theta$ and plot $W$ as a function of $\theta$ and $\phi$. (A) Contour plot of $W$ on the sphere with light and dark regions correspond to excitation and inhibition respectively. (B) Surface plot of $W$ in the $(\theta, \phi)$-plane.

each other whereas those with differing orientation inhibit each other. This is the standard interaction assumption of the ring model $[4,47,50]$. On the other hand, around the pinwheels, all orientations uniformly excite, which is consistent with the fact that although the cells around a pinwheel can differ greatly in their orientation preference, they are physically close together within the hypercolumn.

It is possible to construct a more general form of $\mathbf{O}(3)$-invariant weight distribution using spherical harmonics. Any sufficiently smooth function $f(\theta, \phi)$ on the sphere can be expanded in a uniformly convergent double series of spherical harmonics

$f(\theta, \phi)=\sum_{n=0}^{\infty} \sum_{m=-n}^{n} a_{n m} Y_{n}^{m}(\theta, \phi)$

The functions $Y_{n}^{m}(\theta, \phi)$ constitute the angular part of the solutions of Laplace's equation in three dimensions, and thus form a complete orthonormal set. The orthogonality relation is

$$
\begin{aligned}
& \int_{0}^{\pi} \int_{0}^{\pi} Y_{n_{1}}^{m_{1} *}(\theta, \phi) Y_{n_{2}}^{m_{2}}(\theta, \phi) \frac{\sin \theta \mathrm{d} \theta \mathrm{d} \phi}{2 \pi} \\
& =\frac{1}{4 \pi} \delta_{n_{1}, n_{2}} \delta_{m_{1}, m_{2}} .
\end{aligned}
$$

The spherical harmonics are given explicitly by

$Y_{n}^{m}(\theta, \phi)=(-1)^{m} \sqrt{\frac{2 n+1}{4 \pi} \frac{(n-m) !}{(n+m) !}} P_{n}^{m}(\cos \theta) \mathrm{e}^{2 \mathrm{i} m \phi}$

for $n \geqslant 0$ and $-n \leqslant m \leqslant n$, where $P_{n}^{m}(\cos \theta)$ is an associated Legendre function. (Note that we have adjusted the definition of the spherical harmonics to take into account the fact $\phi$ takes values between 0 and $\pi$.) The action of $\mathbf{S O}(3)$ on $Y_{n}^{m}(\theta, \phi)$ involves $(2 n+1) \times(2 n+1)$ unitary matrices associated with irreducible representations of $S U(2)$ [2]. From the unitarity of these representations, one can construct an $\mathbf{S O}(3)$-invariant weight distribution of the general form

$W\left(P \mid P^{\prime}\right)=4 \pi \sum_{n=0}^{\infty} \widetilde{W}_{n} \sum_{m=-n}^{n} Y_{n}^{m *}\left(\theta^{\prime}, \phi^{\prime}\right) Y_{n}^{m}(\theta, \phi)$ with $\widetilde{W}_{n}$ real. For simplicity, we neglect higher harmonic contributions to $W\left(P \mid P^{\prime}\right)$ by setting $\widetilde{W}_{n}=0$ for $n \geqslant 2$ so that Eq. (3.7) reduces to Eq. (3.3) on rescaling $\widetilde{W}_{1}$.

\section{Anisotropic horizontal connections}

Given that the long range horizontal connections tend to link neurons with similar feature preferences, one can construct an $\mathbf{O}(3)$-invariant long range distribution $W^{\Delta}$ of the form

$$
\begin{aligned}
W^{\Delta}\left(P \mid P^{\prime}\right)= & H\left[\cos \theta \cos \theta^{\prime}-\sin \theta \sin \theta^{\prime}\right. \\
& \left.\times \cos \left(2\left[\phi-\phi^{\prime}\right]\right)-\cos \alpha\right],
\end{aligned}
$$

where $H[x]=1$ if $x \geqslant 0$ and is zero otherwise. The angle $\alpha$ determines the degree of similarity in the orientation and spatial frequency preference of the linked cells, and hence the patch size. It follows that the distribution (2.4) is invariant under the action of the group $\Gamma_{\mathcal{L}} \times \mathbf{O}(3)$ where $\Gamma_{\mathcal{L}}$ is the discrete symmetry group of the lattice $\mathcal{L}$. However, it is likely that in two dimensions the pattern of patchy connections is more complicated than this.

For example, recent optical imaging experiments combined with anatomical tracer injections suggest that there is a spatial anisotropy in the distribution of patchy horizontal connections, as illustrated in Fig. 11. It will be seen from the left panel of Fig. 11 that the anisotropy is particularly pronounced in the tree shrew, where differing iso-orientation patches preferentially connect to neighboring patches in such a way as to form continuous contours following the topography of the retinocortical map. That is, the major axis of the horizontal connections tends to run parallel to the visuotopic axis of the connected cells' common orientation preference. There is also a clear anisotropy in the patchy connections of primates, as seen in the right panel. However, in this case most of the anisotropy can be accounted for by the fact that there is a stretching in the direction orthogonal to ocular dominance columns $[1,48]$. It is possible that when this stretching is factored out, there remains a weak anisotropy correlated with orientation 

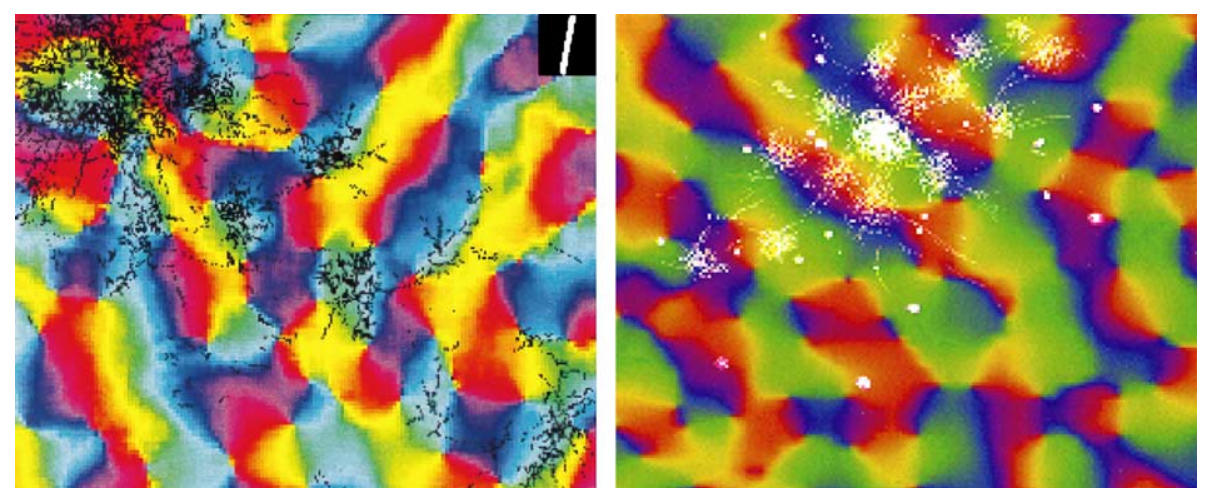

Fig. 11. Lateral Connections made by V1 cells in Tree Shrew (Left panel) and Owl Monkey (Right panel) V1. A radioactive tracer is used to show the locations of all terminating axons from cells in a central injection site, superimposed on an orientation map obtained by optical imaging. Redrawn from $[9,48]$.

selectivity but this remains to be confirmed experimentally. The possible functional role of this anisotropy has been a major focus of our recent work on the dynamics of orientation tuning in V1 $[10,15,16]$.

Anisotropy in the horizontal connections can be incorporated into the coupled lattice model (2.2) by modifying the weight distribution (2.4) along the following lines:

$$
\begin{aligned}
w\left(\ell, P \mid \ell^{\prime}, P^{\prime}\right)= & \delta_{\ell, \ell^{\prime}} W\left(P \mid P^{\prime}\right)+\beta J^{\Delta}\left(\left|\ell-\ell^{\prime}\right|\right) \\
& \times W^{\Delta}\left(P \mid P^{\prime}\right) \mathcal{A}_{\eta}\left(\phi-\psi_{\ell, \ell^{\prime}}\right)
\end{aligned}
$$

with $\psi_{\ell, \ell^{\prime}}=\arg \left(\ell-\ell^{\prime}\right), \quad \mathcal{A}_{\eta}(\phi)=H(\eta-|\phi|)+H(\eta-$ $|\phi+\pi|)$ and $W^{\Delta}$ given by Eq. (4.1). The parameter $\eta$ determines the degree of anisotropy, that is the angular spread of the horizontal connections around the axis joining cells with similar orientation preferences. An elegant feature of the spherical model is that it naturally incorporates the fact that, at the population level, there is zero selectivity for orientation at the pinwheels. In other words, the solution $a(\ell, \theta, \phi)$ expanded in terms of spherical harmonics is independent of $\phi$ at $\theta=0, \pi$. This implies that the lateral weight distribution (4.2) has to be isotropic at the pinwheels. In order to incorporate any anisotropy away from the pinwheels, we conclude that the spread parameter has to be $\theta$-dependent, $\eta=\eta(\theta)$ with $\eta(0)=\eta(\pi)=\pi / 2$. This is illustrated in Fig. 12. It is also possible that the coupling parameter $\beta$ is itself spatial frequency dependent. There is recent experimental evidence indicating that some cells located outside the $\mathrm{CO}$ blobs have very little in the way of lateral connections [59], thus leading to an effective reduction in connectivity at the population level. Since the CO blobs have a strong association with the orientation singularities corresponding to low spatial frequencies $[32,36]$ the coupling may be larger around the low frequency pinwheels.

An interesting mathematical property of the anisotropic weight distribution (4.2) is that it reduces the symmetry group from $\Gamma_{\mathcal{L}} \times \mathbf{O}(3)$ to $\mathbf{T}_{\mathcal{L}}+\mathbf{D}_{n}$ where $\mathbf{T}_{\mathcal{L}}$

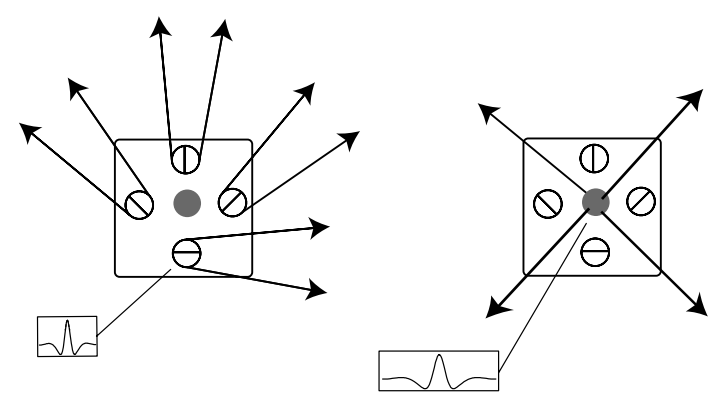

Fig. 12. Cells at intermediate spatial frequencies send out horizontal connections to cells in other hypercolumns in a direction parallel to their common preferred orientation, whereas cells within blob regions (at low spatial frequency pinwheels) connect to other blob regions in an isotropic fashion (similarly for high spatial frequency pinwheels).

denotes the group of lattice translations and $\mathbf{D}_{n}, n=2,4$ or 6, is the lattice holohedry consisting of the set of discrete rotations and reflections that preserve the lattice (see Fig. 13). The associated group action is

$$
\begin{aligned}
& \ell_{s} \cdot(\ell, \theta, \phi)=\left(\ell+\ell_{s}, \theta, \phi\right), \quad \ell_{s} \in \mathbf{T}_{\mathcal{L}}, \\
& \xi \cdot(\ell, \theta, \phi)=\left(R_{\xi} \ell, \theta, \phi+\xi\right), \\
& \kappa \cdot(\ell, \theta, \phi)=\left(R_{\kappa} \ell, \theta,-\phi\right),
\end{aligned}
$$

where $(\xi, \kappa) \in D_{n}, R_{\xi}$ denotes the planar rotation through an angle $\xi$ and $R_{\kappa}$ denotes the reflection $\left(x_{1}, x_{2}\right) \mapsto\left(x_{1},-x_{2}\right)$. The corresponding group action on a function $a: \mathcal{L} \times S^{2} \rightarrow \mathbf{R}$ is given by
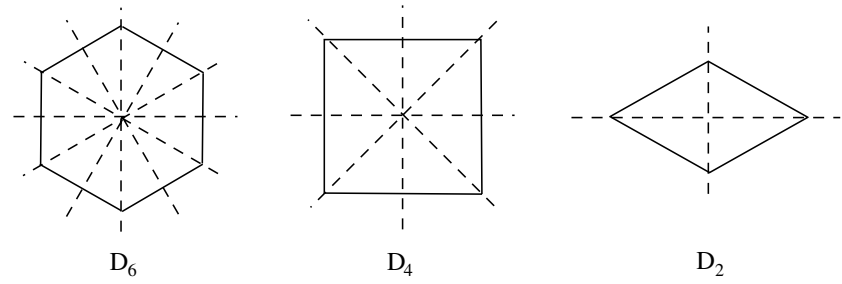

Fig. 13. Holohedries of the plane. 
$\gamma \cdot a(\ell, P)=a\left(\gamma^{-1} \cdot(\ell, P)\right) \quad$ for all $\gamma \in \mathbf{T}_{\mathcal{L}} \dot{+} \mathbf{D}_{n}$

and the invariance of $w_{\text {lat }}\left(\mathbf{r}, P \mid \mathbf{r}^{\prime}, P^{\prime}\right)$ is expressed as

$$
\begin{aligned}
\gamma \cdot w\left(\ell, P \mid \ell^{\prime}, P^{\prime}\right) & =w\left(\gamma^{-1} \cdot(\ell, P) \mid \gamma^{-1} \cdot\left(\ell^{\prime}, P^{\prime}\right)\right) \\
& =w\left(\ell, P \mid \ell^{\prime}, P^{\prime}\right) .
\end{aligned}
$$

It can be seen that the rotation operation comprises a translation or shift of the orientation preference label $\phi$ to $\phi+\xi$, together with a rotation or twist of the position vector $\ell$ by the angle $\xi$. The spatial frequency is not affected by rotations. The fact that the weight distribution is invariant with respect to this shift-twist action has important consequences for the global dynamics of $\mathrm{V} 1$ in the presence of anisotropic horizontal connections $[10,15]$.

\section{Cortical pattern formation}

Suppose, for concreteness, that there is a time-independent external bias $h$ such that $a(\ell, P, t)=a_{0}$ is a fixed point solution of Eq. (2.2). Setting $a(\ell, P, t)=a_{0}+$ $\mathrm{e}^{\lambda t} a(\ell, P)$ and linearizing about the fixed point leads to the eigenvalue equation

$$
\begin{aligned}
\lambda a(\ell, P)= & -\alpha a(\ell, P) \\
& +\mu \int_{0}^{\pi} \int_{0}^{\pi} \sum_{\ell^{\prime} \in \mathcal{L}} w\left(\ell, P \mid \ell^{\prime}, P^{\prime}\right) a\left(\ell^{\prime}, P^{\prime}\right) D P^{\prime}
\end{aligned}
$$

with $P=(\theta, \phi), P^{\prime}=\left(\theta^{\prime}, \phi^{\prime}\right)$ and $D P^{\prime}=\sin \theta^{\prime} \mathrm{d} \theta^{\prime} \mathrm{d} \phi^{\prime} / 4 \pi$. Since the weight distribution $w$ is bounded, it follows that when the network is in a low activity state $a_{0}$ such that $\mu=\sigma^{\prime}\left(a_{0}\right) \approx 0$, any solution of Eq. (5.1) satisfies $\operatorname{Re} \lambda<0$ and the fixed point is linearly stable. However, when the excitability of the network is increased, either through the action of some hallucinogen or through external stimulation, $\mu$ increases. This can induce a
Turing instability leading to the formation of spontaneous cortical activity patterns. In this section we derive conditions for the onset of a Turing instability, and discuss the nature of the resulting patterns. We do not address the selection and stability of the patterns here: a detailed review of weakly nonlinear analysis and amplitude equation methods is presented elsewhere [12].

Let us first consider the case of isotropic and homogeneous long-range connections. Substitution of Eqs. (2.4), (3.3) and (4.1) into Eq. (5.1) gives

$$
\begin{aligned}
{\left[\frac{\lambda+\alpha}{\mu}\right] a(\ell, P)=} & \int_{0}^{\pi} \int_{0}^{\pi} W\left(P \mid P^{\prime}\right) a\left(\ell, P^{\prime}\right) D P^{\prime} \\
& +\beta \sum_{\ell^{\prime} \neq \ell} J^{\Delta}\left(\left|\ell-\ell^{\prime}\right|\right) \\
& \times \int_{0}^{\pi} \int_{0}^{\pi} W^{\Delta}\left(P \mid P^{\prime}\right) a\left(\ell^{\prime}, P^{\prime}\right) D P^{\prime} .
\end{aligned}
$$

Since the weight distribution $w$ is invariant under the action of $\Gamma_{\mathcal{L}} \times \mathbf{O}(3)$, it follows that the eigensolutions are of the form

$a(\ell, P)=\mathrm{e}^{\mathrm{ik} \cdot \ell} Y_{n}^{m}(\theta, \phi)$

for $n \in \mathbf{Z},-n \leqslant m \leqslant n$ and $\mathbf{k} \in \widehat{\Lambda} \subset \widehat{\mathcal{L}}$. Here $\mathbf{k}$ is restricted to the first Brillouin zone $\widehat{\Lambda}$ of the reciprocal lattice $\widehat{\mathcal{L}}$ [3]. The first Brillouin zone is the fundamental domain around the origin of the reciprocal lattice formed by the perpendicular bisectors of the shortest lattice vectors (of length $2 \pi / L$ ). Examples for the square and hexagonal lattices are shown in Fig. 14. The corresponding eigenvalue $\lambda=\lambda_{n}(\mathbf{k})$ is $(2 n+1)$-fold degenerate such that

$\lambda_{n}(\mathbf{k})=-\alpha+\mu\left[\widetilde{W}_{n}+\beta \widetilde{J}^{\Delta}(\mathbf{k}) \widetilde{W}_{n}^{\Delta}\right]$,

where $\widetilde{W}_{n}$ and $\widetilde{W}_{n}^{\Delta}$ are the $n$th coefficients in the spherical harmonic expansions of $W$ and $W^{\Delta}$ (see Eq. (3.7)) and
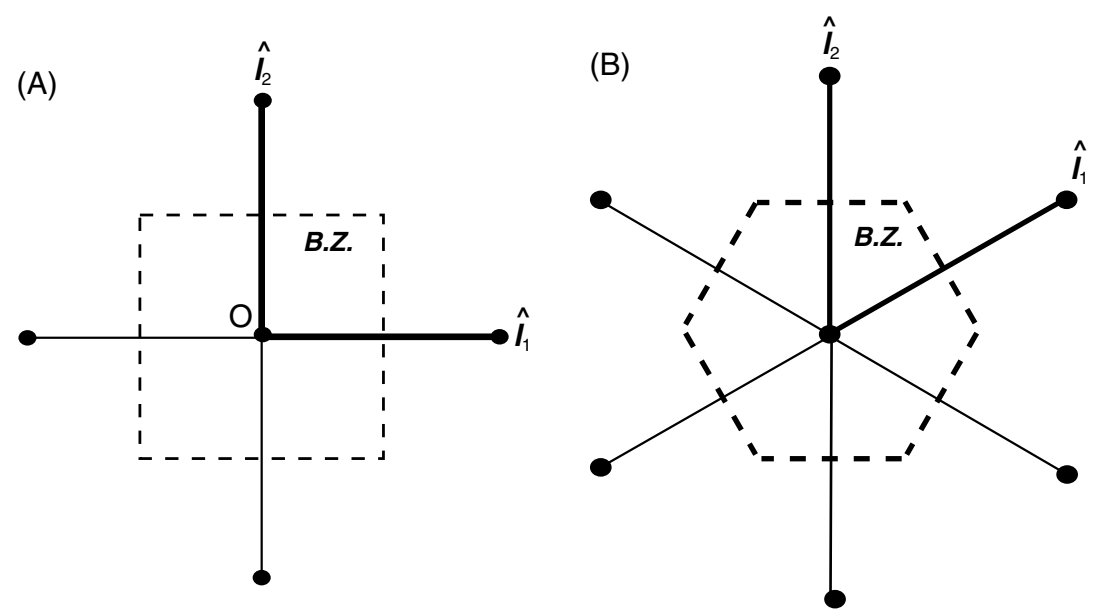

Fig. 14. Construction of the first Brillouin zone in (A) the reciprocal square lattice and (B) the reciprocal hexagonal lattice. 
$J^{\Delta}(\ell)=\frac{L^{2}}{4 \pi^{2}} \int_{\widehat{A}} \widetilde{J}^{\Delta}(\mathbf{k}) \mathrm{e}^{\mathrm{i} \mathbf{k} \cdot \ell} \mathrm{d} \mathbf{k}$,

$\widetilde{J}^{\Delta}(\mathbf{k})=\sum_{\ell \in \mathcal{L}} J^{\Delta}(\ell) \mathrm{e}^{-\mathrm{ik} \cdot \ell}$.

In the limiting case $\beta=0$, each isolated hypercolumn is described by the spherical model $[13,14]$ :

$$
\begin{aligned}
\frac{\partial a(\ell, P, t)}{\partial t}= & -\alpha a(\ell, P, t) \\
& +\int_{0}^{\pi} \int_{0}^{\pi} w\left(P \mid P^{\prime}\right) \sigma\left(a\left(\ell, P^{\prime}, t\right)\right) D P^{\prime}, \quad \ell \in \mathcal{L} .
\end{aligned}
$$

The eigenvalues $\Lambda_{n}$ are now k-independent. Assuming that $\widetilde{W}_{1}>\widetilde{W}_{n}$ for all $n \neq 1$, the condition for marginal stability reduces to $\mu_{c}=\alpha / \widetilde{W}_{1}$. It can be shown that there exists an $\mathbf{O}(3)$-invariant submanifold of marginally stable states involving linear combinations of the three first order spherical harmonics [13]:

$a(\ell, \theta, \phi)=\sum_{m=0, \pm 1} z_{m}(\ell) Y_{1}^{m}(\theta, \phi)$

with $z_{-1}(\ell)=z_{1}^{*}(\ell)$ and $2 z_{-1} z_{1}+z_{0}$ independent of $\ell$. It follows that the eigenmodes can be rewritten in the form

$$
\begin{aligned}
a(\ell, \theta, \phi)= & A[\cos \theta \cos \bar{\theta}(\ell)+\sin \theta \sin \bar{\theta}(\ell) \\
& \times \cos (2[\phi-\bar{\phi}(\ell)])] \\
= & A \sum_{m=0, \pm 1} f_{m}(\theta, \phi) f_{m}(\bar{\theta}(\ell), \bar{\phi}(\ell))
\end{aligned}
$$

with

$f_{0}(\theta, \phi)=\cos \theta$,

$f_{1}(\theta, \phi)=\sin \theta \cos 2 \phi$,

$f_{-1}=\sin \theta \sin 2 \phi$.

This solution represents a tuning surface for orientation and spatial frequency with a solitary peak located at $(\bar{\theta}(\ell), \bar{\phi}(\ell))[13]$. In Fig. 15 we show some typical tuning surfaces obtained by numerically solving the rate equation (5.6) for fixed $\ell$ and projecting the solution onto the $(p, \phi)$-plane. Note that the tuning surface broadens when the peak response is shifted towards either the high or low spatial frequency pinwheel, reflecting the reduction in orientation selectivity in these regions [14].

If the horizontal connections are now switched on, there is a k-dependent splitting of the degenerate eigenvalue $\lambda_{1}$. Since, the long range connections are narrowly tuned with respect to orientation and spatial frequency, the corresponding spherical harmonic coefficients $\widetilde{W}_{n}^{\Delta}$ are only weakly dependent on $n \in \mathbf{Z}$. This implies that the horizontal connections do not excite other spherical harmonic components within a hypercolumn, and the condition for marginal stability of the homogeneous fixed point is obtained from the eigenvalue equation
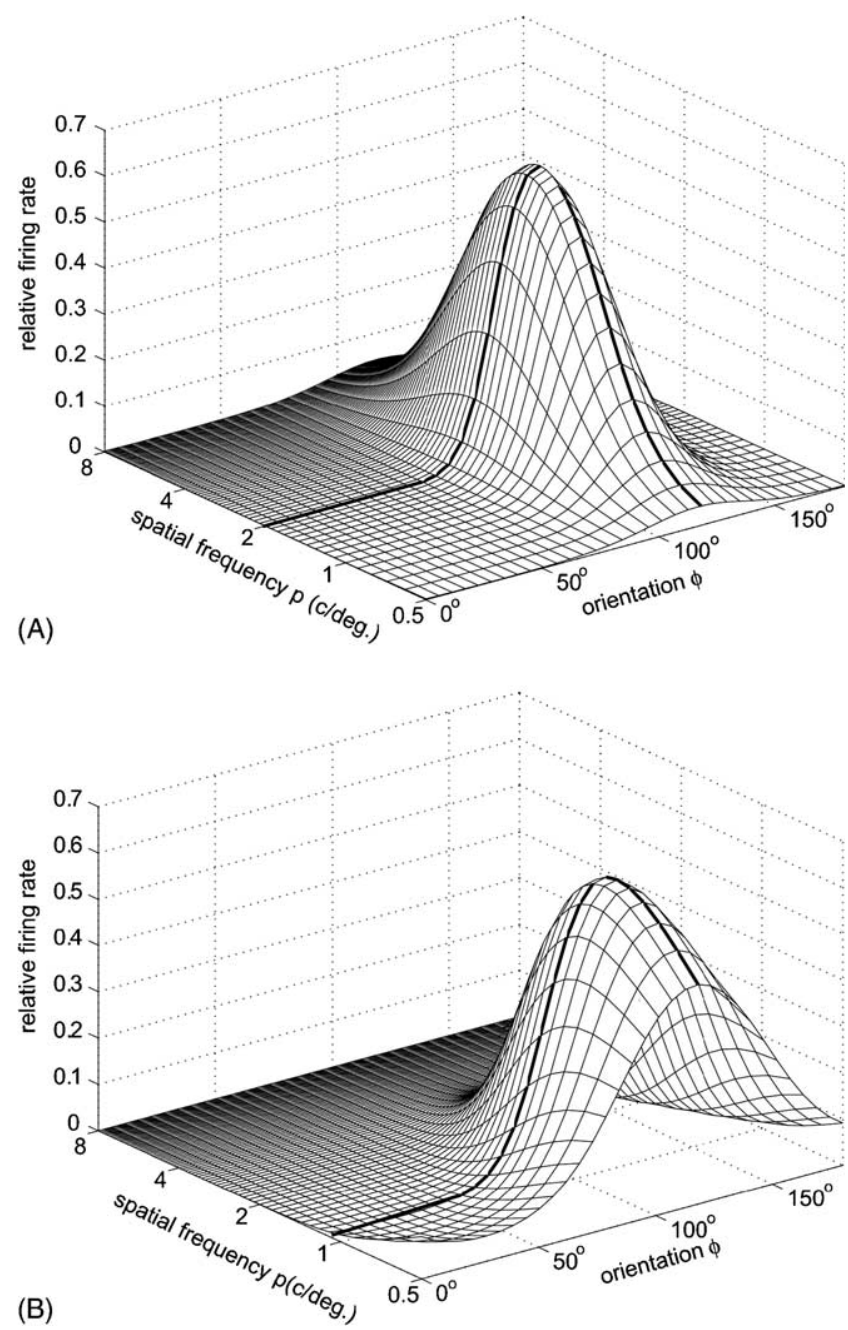

Fig. 15. Tuning surfaces in the $\{p, \phi\}$ plane for the spherical model of a hypercolumn. Weight distribution is given by Eq. (3.3) with $\widetilde{W}_{0}=-1$ and $\widetilde{W}_{1}=1$. The parameters of the compressive nonlinearity $\mathscr{Q}$ in Eq. (3.1) are $\delta=1.5$ and $p_{0}=2 c /$ deg. (A) $\bar{\theta}=\pi / 2$ and $\bar{\phi}=\pi / 2$, (B) $\bar{\theta}=\pi / 4$ and $\bar{\phi}=\pi / 2$.

$\frac{\lambda_{1}(\mathbf{k})+\alpha}{\mu}=\frac{\alpha}{\mu_{c}}+\beta \widetilde{J}^{\Delta}(\mathbf{k})$,

where a positive factor $\widetilde{W}_{1}^{\Delta}$ has been absorbed into $\beta$. Suppose that $\widetilde{J}^{\Delta}(\mathbf{k})$ has a minimum at $\mathbf{k}=\mathbf{k}_{c}$ when $\beta<0$. The homogeneous fixed point is then marginally stable at the critical value

$\widehat{\mu}_{c}=\frac{\alpha \mu_{c}}{\alpha+\beta \mu_{c} \widetilde{J}^{\Delta}\left(\mathbf{k}_{c}\right)}$.

Since $\widetilde{J}^{\Delta}(\mathbf{k})$ is invariant with respect to the corresponding lattice holohedry $\mathbf{D}_{n}$, all other wave vectors related to $\mathbf{k}_{c}$ by a discrete rotation will also be selected. The marginally stable eigenmodes will thus be of the form

$a(\ell, \theta, \phi)=A(\ell) \sum_{m=0, \pm 1} f_{m}(\theta, \phi) f_{m}(\bar{\theta}, \bar{\phi})$

for arbitrary constant phases $\bar{\theta}, \bar{\phi}$, and 
$A(\ell)=\sum_{j=1, \ldots, N}\left[u_{j} \mathrm{e}^{\mathbf{i k}_{j} \cdot \ell}+\bar{u}_{j} \mathrm{e}^{-\mathbf{i} \mathbf{k}_{j} \cdot \ell}\right]$

where $u_{j}$ is a complex amplitude with conjugate $\bar{u}_{j}$. Here $N=2$ for the square lattice with $\mathbf{k}_{1}=\mathbf{k}_{c}$ and $\mathbf{k}_{2}=R_{\pi / 2} \mathbf{k}_{c}$, where $R_{\xi}$ denotes rotation through an angle $\xi$. Similarly, $N=3$ for the hexagonal lattice with $\mathbf{k}_{1}=\mathbf{k}_{c}, \mathbf{k}_{2}=R_{2 \pi / 3} \mathbf{k}_{c}$ and $\mathbf{k}_{3}=R_{4 \pi / 3} \mathbf{k}_{c}$.

As an illustrative example, consider a long range distance function for the square lattice that satisfies

$$
\begin{aligned}
J^{\Delta}\left(\left|m \ell_{1}+n \ell_{2}\right|\right)= & \delta_{m, \pm 1} \delta_{n, 0}+\delta_{m, 0} \delta_{n, \pm 1}+A_{1} \delta_{m, \pm 1} \delta_{n, \pm 1} \\
& +A_{2}\left[\delta_{m, \pm 2} \delta_{n, 0}+\delta_{m, 0} \delta_{n, \pm 2}\right]
\end{aligned}
$$

with $A_{2}<A_{1}<1$. The corresponding Fourier transform is

$$
\begin{aligned}
\widetilde{J}^{\Delta}(\mathbf{k})= & 2\left(\cos k_{x}+\cos k_{y}+A_{1}\left[\cos \left(k_{x}+k_{y}\right)\right.\right. \\
& \left.\left.+\cos \left(k_{x}-k_{y}\right)\right]+A_{2}\left[\cos 2 k_{x}+\cos 2 k_{y}\right]\right)
\end{aligned}
$$

for $\mathbf{k}=\left(k_{x}, k_{y}\right)$. Contour plots of $\widetilde{J}^{\Delta}(\mathbf{k})$ as a function of $\mathbf{k}$ are shown in Fig. 16 with $\mathbf{k}$ restricted to lie in the first Brillouin zone. One can see that there is a global maximum at $\mathbf{k}=0$ and four global minima, reflecting the four-fold symmetry of the lattice. (Also shown is the corresponding contour plot for nearest neighbour coupling on an hexagonal lattice where there is a six-fold symmetry). This means that in the excitatory regime $(\beta>0)$ there is a bulk instability with respect to the lattice, but a Turing instability with respect to orientation and spatial frequency. Thus each hypercolumn exhibits a tuning surface with the same peak response. On the other hand, in the inhibitory regime $(\beta<0)$ the marginally stable modes have a critical wave number $\mathbf{k}_{c} \neq 0$, implying that there is a Turing instability with respect to both the internal and external degrees of freedom.

The pattern generated by the eigensolution (5.12) with $\mathbf{k}_{c} \neq 0$ consists of a distribution of tuning surfaces across cortex whose peak response alternates between the points $(\bar{\theta}, \bar{\phi})$ and $(\pi-\bar{\theta}, \bar{\phi}+\pi / 2)$ according to the sign of the amplitude $A(\ell)$. For wave vectors $\mathbf{k}_{c}$ that are commensurate with the lattice such alternations in sign generate a periodic tiling of the cortical plane consisting of stripes, hexagons or squares, whereas incommensurate wave vectors generate quasiperiodic patterns. Interestingly, if one highlights those regions of cortex that correspond to high levels of activity one obtains patterns
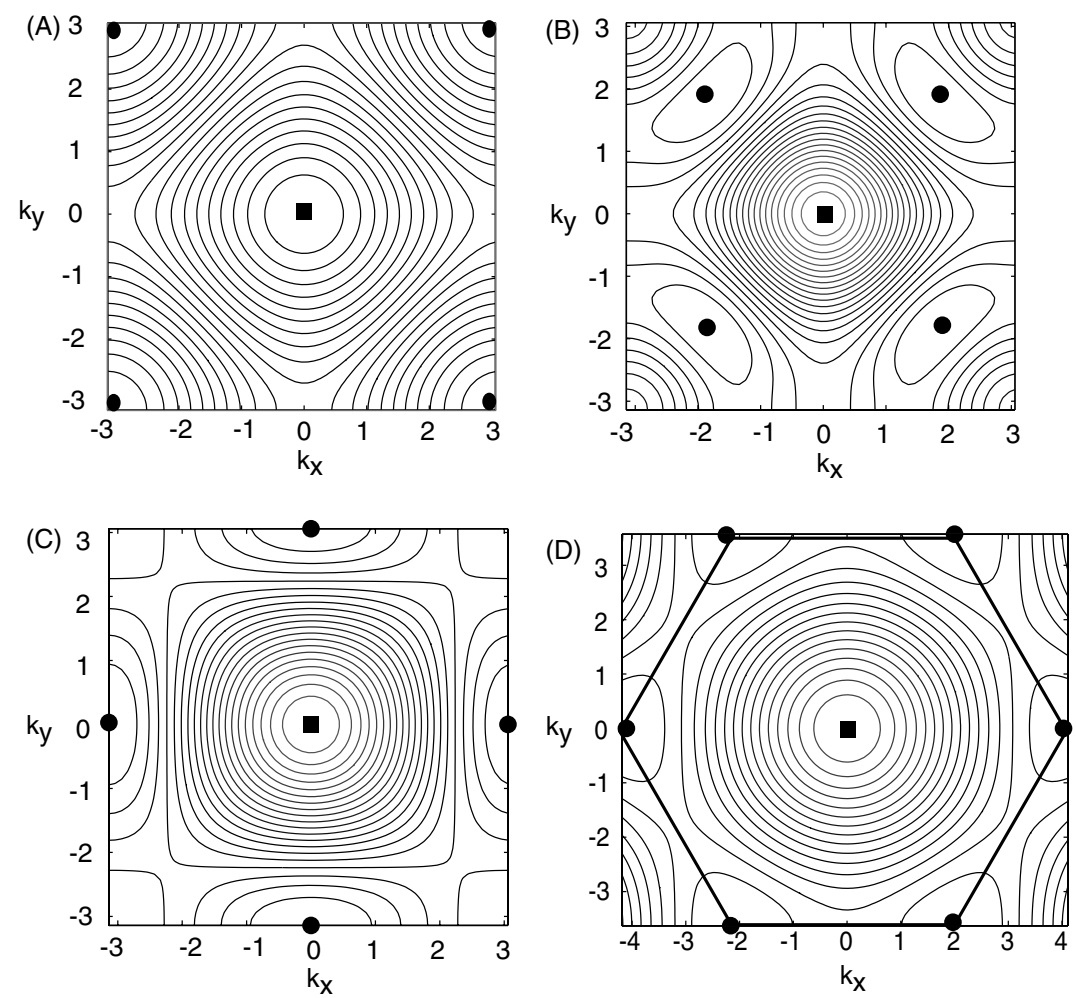

Fig. 16. (A-C) Contour plot of $\widetilde{J}^{\Delta}(\mathbf{k})$ satisfying Eq. (5.15) for $\mathbf{k}=\left(k_{x}, k_{y}\right)$ in the first Brillouin zone of the square lattice. Filled circles indicate locations of global minima whereas filled square indicates location of global maximum at origin. (A) $A_{1}=A_{2}=0$ with four minima at $\left(k_{x}, k_{y}\right)=( \pm \pi, \pm \pi)$; (B) $A_{1}=0.8$ and $A_{1}=0.6$ with four minima inside the Brillouin zone boundary; (C) $A_{1}=0.8, A_{2}=0$ with four minima at $\left(k_{x}, k_{y}\right)=( \pm \pi, 0)$ and $\left(k_{x}, k_{y}\right)=(0, \pm \pi)$; (D) Contour plot of $\widetilde{J}^{\Delta}(\mathbf{k})$ for nearest neighbour coupling on the hexagonal lattice showing a six-fold symmetry. 
reminiscent of $2 \mathrm{DG}$ images. For example, if $\bar{\theta}=\pi / 2$ then the maximal response of each hypercolumn occurs at $\theta=\pi / 2$ (intermediate spatial frequencies) and the peak orientation alternates between $\bar{\phi}$ and the orthogonal orientation $\bar{\phi}+\pi / 2$. One would then predict that for intermediate spatial frequencies activity hotspots would predominantly lie in the linear zones, just as in Fig. 2A. On the other hand, if $\bar{\theta}=0, \pi$ then the response of each hypercolumn is approximately independent of orientation preference and the maximal response alternates between high and low spatial frequencies. Thus one would expect the hotspots to coincide with either high or low spatial frequency pinwheels, i.e. about $50 \%$ should line up with CO blobs.

It turns out that if one allows for some anisotropy in the horizontal connections along the lines of Eq. (4.2), then the solutions that bifurcate from the homogeneous fixed point fall into one of three distinct classes [12]:

(i) Odd oriented patterns

$$
\begin{aligned}
a(\ell, \theta, \phi)= & \sin \theta \sum_{j=1, \ldots, N}\left[u_{j} \mathrm{e}^{\mathrm{i} \mathbf{k}_{j} \cdot \ell}+\bar{u}_{j} \mathrm{e}^{-\mathrm{i} \mathbf{k}_{j} \cdot \ell}\right] \\
& \times \sin \left(2\left[\phi-\zeta_{j}\right]\right) .
\end{aligned}
$$

Each hypercolumn has a peak response at an intermediate spatial frequency and at an orientation that is determined by a linear combination of plane waves modulated by the odd $\pi$-periodic function $\sin \left(2\left[\phi-\zeta_{j}\right]\right)$ with $\zeta_{j}$ the direction of the corresponding wave vector, $\mathbf{k}_{j}=k_{c}\left(\cos \zeta_{j}, \sin \zeta_{j}\right)$.

(ii) Even oriented patterns

$$
\begin{aligned}
a(\ell, \theta, \phi)= & \sin \theta \sum_{j=1, \ldots, N}\left[u_{j} \mathrm{e}^{\mathbf{i} \mathbf{k}_{j} \cdot \ell}+\bar{u}_{j} \mathrm{e}^{-\mathbf{i} \mathbf{k}_{j} \cdot \ell}\right] \\
& \times \cos \left(2\left[\phi-\zeta_{j}\right]\right) .
\end{aligned}
$$

Each hypercolumn has a peak response at an intermediate spatial frequency and at an orientation that is determined by a linear combination of plane waves modulated by the even $\pi$-periodic function $\cos \left(2\left[\phi-\zeta_{j}\right]\right)$.

(iii) Non-oriented patterns

$$
a(\ell, \theta, \phi)=\cos \theta \sum_{j=1, \ldots, N}\left[u_{j} \mathrm{e}^{\mathrm{i} \mathbf{k}_{j} \cdot \ell}+\bar{u}_{j} \mathrm{e}^{-\mathbf{i} \mathbf{k}_{j} \cdot \ell}\right]
$$

in which the peak response alternates between low and high spatial frequency pinwheels.

The existence of these three types of solution reflects the explicit breaking of $\mathbf{O}(3)$ symmetry, which induces a splitting of the degenerate eigenmodes associated with the three first-order spherical harmonics.

\section{The retino-cortical map}

Any spontaneously generated or stimulus-evoked cortical activity pattern in V1 maps to a corresponding real or hallucinatory image on the retina. Elsewhere we have reconstructed such images in the case of the odd and even oriented patterns given by Eqs. (5.16) and (5.17) [10]. These patterns were first reduced to vector fields on the cortical lattice $\mathcal{L}$ by determining the orientation at which each hypercolumn had its maximal response. The vector fields were then mapped back into visual space under the inverse retino-cortical map, leading to contoured images that reproduced a number of common hallucinations. It is currently less clear how to incorporate spatial frequency into this reconstruction, although it is likely to generate textured as well as contoured images. Here we shall restrict our discussion to the simpler problem of how the retinal labels for position, orientation and spatial frequency preference all transform under the action of the retino-cortical map. We approach this problem by considering the distribution of receptive field profiles across $\mathrm{V} 1$, and then show how dilatation and rotation of a retinal image is equivalent to translation of the corresponding activity pattern in V1.

Assume that, for a single neuron, stimulus feature preferences arise due to a weak feedforward bias in its receptive field. A reasonable model of the two-dimensional receptive field of a simple $\mathrm{V} 1$ neuron (in retinal coordinates $\mathbf{R}=\{X, Y\})$ is the difference of Gaussians

$$
\begin{aligned}
U_{0}(\mathbf{R})= & \frac{\sqrt{\kappa}}{2 \pi \sigma_{+}} \exp \left[-\frac{1}{2 \sigma_{+}^{2}}\left(\kappa^{2} X^{2}+Y^{2}\right)\right] \\
& -\frac{\alpha}{2 \pi \sigma_{-}} \exp \left[-\frac{1}{2 \sigma_{-}^{2}}\left(X^{2}+Y^{2}\right)\right] .
\end{aligned}
$$

This represents a center-surround profile in which the excitatory center is an ellipse with eccentricity $\kappa>1$ whose major axis runs along the $Y$-direction. The inhibitory surround is taken to be circular but with a larger half width, $\sigma_{-}>\sigma_{+}$. Taking the two dimensional Fourier transform of $u$ shows that

$$
\begin{aligned}
\widetilde{U}_{0}(\mathbf{q})= & \exp \left[-\frac{\sigma_{+}^{2} q^{2}}{2}\left(\kappa^{-2} \cos ^{2} \varphi+\sin ^{2} \varphi\right)\right] \\
& -\alpha \exp \left[-\frac{\sigma_{-}^{2} q^{2}}{2}\right]
\end{aligned}
$$

for $\mathbf{q}=\{q, \varphi\}$ in polar coordinates. The function $\widetilde{U}_{0}(\mathbf{q})$ has a maximum at $\mathbf{p}=\{p, \phi\}$ with $\phi=0$ and

$p=\sqrt{\frac{4}{\sigma_{-}^{2}-\kappa^{-2} \sigma_{+}^{2}} \ln \left[\frac{\sqrt{\alpha} \kappa \sigma_{-}}{\sigma_{+}}\right]}$.

Setting $\sigma_{+}=\sigma$ and $\sigma_{-}=\hat{\kappa} \sigma$ and taking $\hat{\kappa}, \kappa, \alpha$ to be fixed, it follows that the spatial frequency $p$ is inversely proportional to the size $\sigma$ of the receptive field, 
$p=\frac{A}{\sigma}, \quad A=\sqrt{\frac{4}{\hat{\kappa}^{2}-\kappa^{-2}} \ln [\sqrt{\alpha} \kappa \hat{\kappa}]}$

and we can rewrite $U_{0}$ as

$$
\begin{aligned}
U_{0}(\mathbf{R}, p)= & \frac{p \sqrt{\kappa}}{2 \pi A} \exp \left[-\frac{p^{2}}{2 A^{2}}\left(\kappa^{2} X^{2}+Y^{2}\right)\right] \\
& -\frac{\alpha p}{2 \pi \hat{\kappa} A} \exp \left[-\frac{p^{2}}{2 A^{2} \hat{\kappa}^{2}}\left(X^{2}+Y^{2}\right)\right] .
\end{aligned}
$$

We then define

$$
U(\mathbf{R} \mid \overline{\mathbf{R}}, \mathbf{p})=U_{0}\left(T_{\phi}[\mathbf{R}-\overline{\mathbf{R}}], p\right)
$$

to represent the receptive field profile of a V1 neuron centered at the retinal coordinate $\overline{\mathbf{R}}=\{\bar{X}, \bar{Y}\}$ with orientation preference $\phi$ and spatial frequency preference $p$, and

$T_{\phi}=\left(\begin{array}{cc}\cos \phi & -\sin \phi \\ \sin \phi & \cos \phi\end{array}\right)$.

Given a visual stimulus with intensity $I(\mathbf{R})$, the effective input to such a neuron will be of the form

$h(\overline{\mathbf{R}}, \mathbf{p})=\int I(\mathbf{R}) U(\mathbf{R} \mid \overline{\mathbf{R}}, \mathbf{p}) \mathrm{d} \mathbf{R}$.

We now make the following ansatz: given a neuron with orientation preference $\phi$, spatial frequency preference $p$ and receptive field center $\overline{\mathbf{R}}$, there exists a family of receptive fields generated by the action of dilatations and rotations on the receptive field of the given neuron. This ansatz is motivated by the idea that the representation of images can be considered in terms of the cross-correlation of an image with a template or filter that is equivalent to a receptive field profile [45]. That is, given an image in the visual field $I(\mathbf{R})$ and a receptive field profile $U(\mathbf{R} \mid \overline{\mathbf{R}}, \mathbf{p})$, transformed via some symmetry group $G$, then the cross-correlation of $I$ and $u$ with respect to $g \in G$ is

$h_{g}(\overline{\mathbf{R}}, \mathbf{p})=\int I(\mathbf{R}) U\left(g^{-1} \mathbf{R} \mid \overline{\mathbf{R}}, \mathbf{p}\right) \mathrm{d}^{2} \mathbf{R}$.

Thus, let $G$ be the group of dilatations $\mathbf{R} \rightarrow a \mathbf{R}$ and rotations $\mathbf{r} \rightarrow T_{\psi} \mathbf{r}$ in the retinal plane, where $a>0$ and $T_{\psi} \in S O(2)$ for $0 \leqslant \psi<2 \pi$. It follows from Eq. (6.1) that

$$
\begin{aligned}
U\left(g^{-1} \mathbf{R} \mid \overline{\mathbf{R}}, \mathbf{p}\right) & =U\left(a^{-1} T_{-\psi} \mathbf{R} \mid \overline{\mathbf{R}}, \mathbf{p}\right) \\
& =U\left(\mathbf{R} \mid a T_{\psi} \overline{\mathbf{R}}, a^{-1} T_{\psi} \mathbf{p}\right)
\end{aligned}
$$

which implies

$h_{g}(\overline{\mathbf{R}}, \mathbf{p})=h\left(\overline{\mathbf{R}}^{\prime}, \mathbf{p}^{\prime}\right)$,

where

$\overline{\mathbf{R}} \rightarrow \overline{\mathbf{R}}^{\prime}=a T_{\psi} \overline{\mathbf{R}}$

and

$\mathbf{p} \rightarrow \mathbf{p}^{\prime}=a^{-1} T_{\psi} \mathbf{p}$
The next major observation is that dilatations and rotations in the retina correspond to translations in V1. For there exists a well defined retino-cortical map $\mathcal{F}: \overline{\mathbf{R}} \rightarrow \mathbf{r}$ between the receptive field center $\overline{\mathbf{R}}$ and the location $\mathbf{r}$ of a neuron in cortex. Except for a small region around the fovea, this map can be approximated by the complex logarithm (see Fig. 17). That is, if $\overline{\mathbf{R}}=\{\bar{R}, \bar{\Theta}\}$ in polar coordinates, then $\mathbf{r} \approx\{\log \bar{R}, \bar{\Theta}\}$ in Cartesian coordinates. Evidently if we introduce the complex representation of $\overline{\mathbf{R}}, Z=\bar{R} \exp (\mathrm{i} \bar{\Theta})$, then $z=\log Z=\log \bar{R}+\mathrm{i} \bar{\Theta}=x+\mathrm{i} y$ generates the complex $V 1$ representation. It follows that the action of dilatations and rotations in the retina, Eq. (6.12), induces a corresponding translation in the cortex

$\mathbf{r} \rightarrow \mathbf{r}^{\prime}=\mathcal{F} \circ a T_{\psi} \circ \mathcal{F}^{-1}(\mathbf{r})$

such that

$x^{\prime}=x+\log a, \quad y^{\prime}=y+\psi$.

Moreover, writing Eq. (6.13) in polar coordinates shows that there is a simultaneous shift in orientation and spatial frequency according to

$\phi \rightarrow \phi^{\prime}=\phi+\psi, \quad v \rightarrow v^{\prime}=v-\log a$,

where $v=\log p$. Eqs. (6.15) and (6.16) imply that there is a linear variation in orientation and (log) spatial frequency across V1. Note that the decrease of spatial frequency as one moves away from the fovea is consistent with the observation that receptive fields tend to be larger in the periphery.

Of course, the above picture is distorted at the local level due to the presence of pinwheels. In order to incorporate this aspect, we imagine that the cortex is partitioned into hypercolumns as detailed in Section 2, with a given hypercolumn having a distribution of receptive field properties consistent with its underlying pinwheel structure. We then assume that (on an appropriately coarse-grained spatial scale) the receptive field properties of all other hypercolumns are generated by the action of dilatations and rotations as previously described. It immediately follows that the relative area of feature space covered by each hypercolumn is the same. For example, although the range of spatial frequencies within a hypercolumn is shifted downwards as one moves away from the fovea, the bandwidth remains invariant. It also follows that the cortical labels $\{\phi, v\}$ for orientation and (log) spatial frequency preference are actually defined relative to the retinal coordinate $\overline{\mathbf{R}}$. That is, if $\phi=\phi_{0}$ and $v=v_{0}$ at $\overline{\mathbf{R}}=(1,0)$ then

$\phi=\phi_{0}-\bar{\Theta}, \quad v=v_{0}+\log \bar{R}$

at $\overline{\mathbf{R}}=\{\bar{R}, \bar{\Theta}\}$. This makes the testable prediction that the long range horizontal interactions tend to connect regions of $\mathrm{V} 1$ with the same relative (rather than absolute) orientation and spatial frequency preference. 


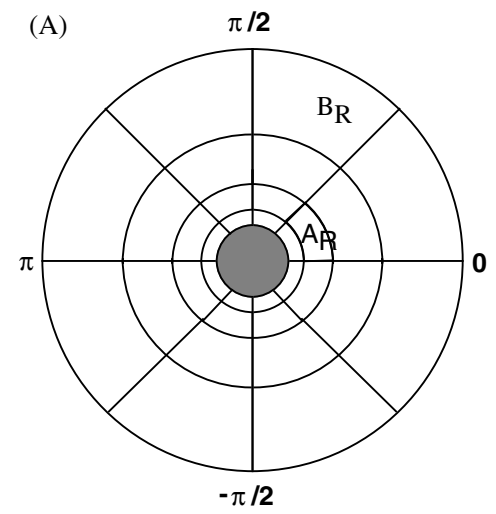

RETINA

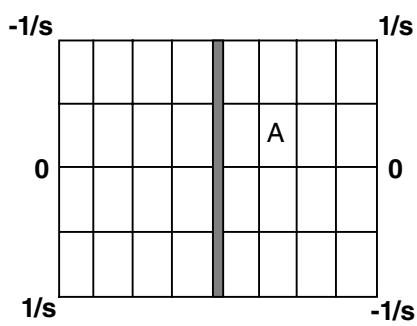

VISUAL CORTEX

(B)
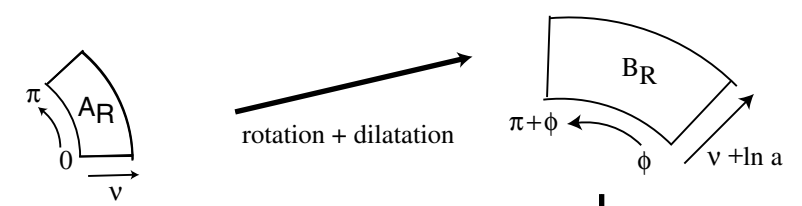

RETINA
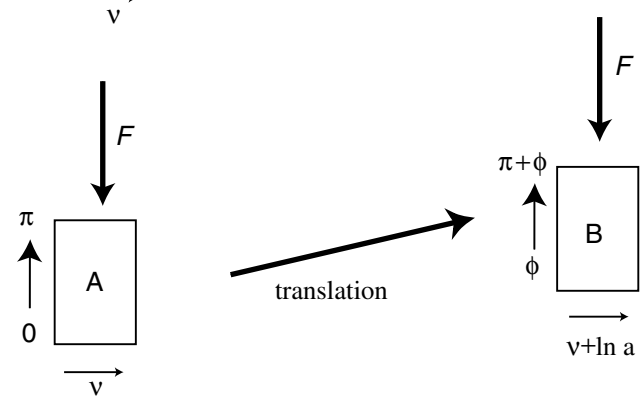

VISUAL CORTEX

Fig. 17. (A) The retino-cortical map generated by the complex logarithm. (B) Action of rotations and dilatations on a local region of visual field that maps to a rectangular region in cortex covering a single hypercolumn.

\section{Discussion}

We conclude from the above analysis that primary visual cortex has a distinctly crystalline-like structure, which is manifested by the distribution of $\mathrm{CO}$ blobs and orientation pinwheels, and by the distribution of patchy horizontal connections. In this paper we have presented a large-scale dynamical model of this periodic structure based on a lattice of interacting hypercolumns. Each hypercolumn is modeled as a network of orientation and spatial frequency selective cells organized around a pair of pinwheels. We have shown that the underlying crystalline structure can have a major effect on the types of spontaneous activity patterns generrated in cortex. In particular, doubly-periodic patterns such as stripes, squares and hexagons can occur through spontaneous symmetry breaking of the associated planar lattice group. Such activity patterns are of particular interest, since they provide an explanation for the occurrence of certain basic types of geometric visual hallucinations. In previous continuum models of cortical pattern formation, the double- periodicty of the solutions was imposed by hand as a mathematical simplification, rather than as a reflection of a real physical lattice $[10,20]$. Thus our new work provides a stronger link between the nature of hallucinatory patterns and the actual structure of cortex. Indeed, we hypothesize that the symmetries and lengthscales of these hallucinatory images are a direct consequence of the geometry of cortical interactions as well as the retino-cortical map.

It is clear that our model involves a number of major simplifications. First, there is some degree of disorder in the distribution of $\mathrm{CO}$ blobs and orientation pinwheels so that it would be more appropriate to consider a disordered rather than an ordered lattice of hypercolumns. Second, we have carried out a phenomenological reduction of the internal structure of each hypercolumn along the lines illustrated in Fig. 7. Such a reduction needs to be carried out in a more rigorous and systematic fashion, in order to fully account for how the local and long-range connections are correlated with the twodimensional orientation and spatial frequency maps. Alternatively, one could consider a continuum model of 
cortex that explicitly incorporates these correlations. A preliminary analysis of such a model suggests that double-periodicity in the feature maps can induce an analog of Bloch waves found in crystalline solids [3], in which activity patterns are localized around $\mathrm{CO}$ blobs or around inter blob regions [11]. Finally, it would be interesting to consider how the periodic structures highlighted in this paper actually develop in cortex, and subsequently influence the development of other cortical structures. Since many models of activity-based cortical development involve some form of pattern forming instability mediated by lateral connections [53], it is likely that some of the techniques familiar in the study of ordered (and disordered) crystalline structures could also be relevant here.

\section{References}

[1] A. Angelucci, J.B. Levitt, E.J.S. Walton, J.-M. Hupe, J. Bullier, J.S. Lund, Circuits for local and global signal integration in primary visual cortex, J. Neurosci. 22 (2002) 8633-8646.

[2] G. Arfken, Mathematical methods for physicists, third ed., Academic Press, San Diego, 1985.

[3] N.W. Ashcroft, N.D. Mermin, Solid state physics, Saunders College Publishing, 1976.

[4] R. Ben-Yishai, R.L. Bar-Or, H. Sompolinsky, Theory of orientation tuning in visual cortex, Proc. Nat. Acad. Sci. 92 (1995) 3844-3848.

[5] G.G. Blasdel, Orientation selectivity, preference, and continuity in monkey striate cortex, J. Neurosci. 12 (1992) 3139-3161.

[6] G.G. Blasdel, G. Salama, Voltage-sensitive dyes reveal a modular organization in monkey striate cortex, Nature 321 (1986) 579-585.

[7] T. Bonhoeffer, A. Grinvald, Orientation columns in cat are organized in pinwheel like patterns, Nature 364 (353) (1991) 429431.

[8] T. Bonhoeffer, D.S. Kim, D. Malonek, D. Shoham, A. Grinvald, Optical Imaging of the layout of functional domains in area 17/18 border in cat visual cortex, European J. Neurosci. 7 (9) (1995) 1973-1988.

[9] W.H. Bosking, Y. Zhang, B. Schofield, D. Fitzpatrick, Orientation selectivity and the arrangement of horizontal connections in tree shrew striate cortex, J. Neurosci. 17 (1997) 2112-2127.

[10] P.C. Bressloff, J.D. Cowan, M. Golubitsky, P.J. Thomas, M. Wiener, Geometric visual hallucinations, Euclidean symmetry and the functional architecture of striate cortex, Phil. Trans. Roy. Soc. Lond. B 356 (2001) 299-330.

[11] P.C. Bressloff, Bloch waves, periodic feature maps and cortical pattern formation, 89 (2002).

[12] P.C. Bressloff, J.D. Cowan, The visual cortex as a crystal, Physica D 173 (2002) 226-258.

[13] P.C. Bressloff, J.D. Cowan, An SO(3) symmetry breaking mechanism for orientation and spatial frequency tuning in visual cortex, Phys. Rev. Lett. 88 (2002) 78102.

[14] P.C. Bressloff, J.D. Cowan, A spherical model of orientation and spatial frequency tuning in a cortical hypercolumn, Phil. Trans. Roy. Soc. B 358 (2003) 1643-1667.

[15] P.C. Bressloff, J.D. Cowan, M. Golubitsky, P.J. Thomas, M. Wiener, What geometric visual hallucinations tell us about the visual cortex, Neural Comput. 14 (2002) 471-492.

[16] P.C. Bressloff, J.D. Cowan, An amplitude equation approach to contextual effects in primary visual cortex, Neural Comput. 14 (2002) 493-525.
[17] R.L. De Valois, K.K. De Valois, Spatial Vision, Oxford University Press, Oxford, 1988.

[18] V. Dragoi, M. Sur, Some properties of recurrent inhibition in primary visual cortex: contrast and orientation dependence on contextual effects, J. Neurophysiol. 83 (2000) 1019-1030.

[19] D.P. Edwards, K.P. Purpura, E. Kaplan, Contrast sensitivity and spatial frequency response of primate cortical neurons in and around the cytochrome oxidase blobs, Vision Res. 35 (1995) 15011523.

[20] G.B. Ermentrout, J.D. Cowan, A mathematical theory of visual hallucination patterns, Biol. Cybernet. 34 (1979) 137-150.

[21] G.B. Ermentrout, Neural networks as spatial pattern forming systems, Rep. Prog. Phys. 61 (1998) 353-430.

[22] C.D. Gilbert, T.N. Wiesel, Clustered intrinsic connections in cat visual cortex, J. Neurosci. 3 (1983) 1116-1133.

[23] C.D. Gilbert, T.N. Wiesel, Columnar specificity of intrinsic horizontal and corticocortical connections in cat visual cortex, J. Neurosci. 9 (1989) 2432-2442.

[24] C.D. Gilbert, Horizontal integration and cortical dynamics, Neuron 9 (1992) 1-13.

[25] J.D. Hirsch, C.D. Gilbert, Synaptic physiology of horizontal connections in the cat's visual cortex, J. Physiol. Lond. 160 (1992) $106-154$.

[26] J.C. Horton, D.H. Hubel, Regular patchy distribution of cytochrome oxidase staining in primary visual cortex of macaque monkey, Nature 292 (1981) 762-764.

[27] J.C. Horton, Cytochrome oxidase patches: a new cytoarchitectonic feature of monkey visual cortex, Phil. Trans. R. Soc. Lond. B 304 (1984) 199-253.

[28] D.H. Hubel, T.N. Wiesel, Receptive fields, binocular interaction and functional architecture in the cat's visual cortex, J. Neurosci. 3 (1962) 1116-1133.

[29] D.H. Hubel, T.N. Wiesel, Receptive fields and functional architecture of monkey striate cortex, J. Physiol. Lond. 195 (1968) $215-243$.

[30] D.H. Hubel, T.N. Wiesel, Uniformity of monkey striate cortex: A parallel relationship between field size, scatter, and magnification factor, J. Comp. Neurol. 158 (1974) 295-306.

[31] D.H. Hubel, T.N. Wiesel, Functional architecture of macaque monkey visual cortex, Proc. Roy. Soc. B 198 (1977) 1-59.

[32] M. Hübener, D. Shoham, A. Grinvald, T. Bonhoeffer, Spatial relationships among three columnar systems in cat area 17 , J. Neurosci. 17 (23) (1997) 9270-9284.

[33] N.P. Issa, C. Trepel, M.P. Stryker, Spatial frequency maps in cat visual cortex, J. Neurosci. 20 (2000) 8504-8514.

[34] S. LeVay, S.B. Nelson, Columnar organization of the visual cortex, in: A.G. Leventhal (Ed.), The Neural Basis of Visual Function, CRC Press, Boca Raton, 1991, pp. 266-315.

[35] Z. Li, Pre-attentive segmentation in the primary visual cortex, Spatial Vision 13 (1999) 25-39.

[36] M.S. Livingstone, D.H. Hubel, Anatomy and physiology of a color system in the primate visual cortex, J. Neurosci. 4 (1984) 309-356.

[37] J.S. Lund, T. Yoshioka, J.B. Levitt, Comparison of intrinsic connectivity in different areas of macaque monkey cerebral cortex, Cerebral Cortex 3 (1993) 148-162.

[38] R. Malach, Y. Amir, M. Harel, A. Grinvald, Relationship between intrinsic connections and functional architecture revealed by optical imaging and in vivo targeted biocytin injections in primate striate cortex, Proc. Natl. Acad. Sci. 90 (1993) 10469 10473.

[39] P.E. Maldonado, I. Gödecke, C.M. Gray, T. Bonhoeffer, Orientation selectivity in pinwheel centers in cat striate cortex, Science 276 (1997) 1551-1555.

[40] J.A. Mazer, W.E. Vinje, J. McDermott, P.H. Schiller, J.L. Gallant, Spatial frequency and orientation tuning dynamics in V1, Proc. Nat. Acad. Sci. 99 (2002) 1645-1650. 
[41] D. McLaughlin, R. Shapley, M. Shelley, D.J. Wielaard, A neuronal network model of macaque primary visual cortex (V1): Orientation tuning and dynamics in the input layer $4 \mathrm{C} \alpha$, Proc. Natl. Acad. Sci. 97 (2000) 8087-8092.

[42] T. Mundel, A. Dimitrov, J.D. Cowan, Visual cortex circuitry and orientation tuning, in: M.C. Mozer, M.I. Jordan, T. Petsche (Eds.), Advances in Neural Information Processing Systems, MIT Press, vol. 9, 1997, pp. 886-893.

[43] K. Murphy, D.G. Jones, R.C.V. Sluyters, Cytochrome oxidase blobs in cat primary visual cortex, J. Neurosci. 15 (1995) 4196-4208.

[44] K. Obermayer, G. Blasdel, Geometry of orientation and ocular dominance columns in monkey striate cortex, J. Neurosci. 13 (1993) 4114-4129.

[45] D.A. Pintsov, Invariant pattern recognition, symmetry, and Radon transforms, J. Opt. Soc. Am. A 6 (1989) 1544-1554.

[46] K.S. Rockland, J. Lund, Intrinsic laminar lattice connections in primate visual cortex, J. Comp. Neurol. 216 (1983) 303-318.

[47] B. Roerig, B. Chen, Relationships of local inhibitory and excitatory circuits to orientation preference maps in ferret visual cortex, Cerebral Cortex 12 (2002) 187-198.

[48] L.C. Sincich, G.G. Blasdel, Oriented axon projections in primary visual cortex of the monkey, J. Neurosci. 21 (2001) 4416-4426.

[49] L.C. Sincich, J.C. Horton, Divided by cytochrome oxidase: A map of the projections from V1 to V2 in macaques, Science 295 (2002) 1734-1737.

[50] D.C. Somers, S. Nelson, M. Sur, An emergent model of orientation selectivity in cat visual cortical simple cells, J. Neurosci. 15 (1995) 5448-5465.
[51] D.C. Somers, E.V. Todorov, A.G. Siapas, L.J. Toth, D.-S. Kim, M. Sur, A local circuit approach to understanding integration of long-range inputs in primary visual cortex, Cerebral Cortex 8 (1998) 204-217.

[52] M. Stetter, H. Bartsch, K. Obermayer, A mean field model for orientation tuning, contrast saturation, and contextual effects in the primary visual cortex, Biol. Cybernet. 87 (2000) 291-304.

[53] N.V. Swindale, The development of topography in visual cortex: a review of models, Network 7 (1996) 161-247.

[54] R.B.H. Tootell, S.L. Hamilton, M.S. Silverman, E. Switkes, R.L. De Valois, Functional anatomy of macaque striate cortex. V. Spatial Frequency, J. Neurosci. 8 (1988) 1610-1624.

[55] M. Tsodyks, T. Kenet, A. Grinvald, A. Arieli, Linking spontaneous activity of single cortical neurons and the underlying functional architecture, Science 286 (1999) 1943-1946.

[56] H.R. Wilson, J.D. Cowan, Excitatory and inhibitory interactions in localized populations of model neurons, Biophys. J. 12 (1972) $1-24$.

[57] H.R. Wilson, J.D. Cowan, A mathematical theory of the functional dynamics of cortical and thalamic nervous tissue, Kybernetik 13 (1973) 55-80.

[58] T. Yoshioka, G.G. Blasdel, J.B. Levitt, J.S. Lund, Relation between patterns of intrinsic lateral connectivity, ocular dominance, and cytochrome oxidase-reactive regions in macaque monkey striate cortex, Cerebral Cortex 6 (1996) 297-310.

[59] N.H. Yabuta, E.M. Callaway, Cytochrome-oxidase blobs and intrinsic horizontal connections of layer $2 / 3$ pyramidal neurons in primate V1, Vis. Neurosci. 15 (1998) 1007-1027. 